\title{
Impact of jet veto resummation on slepton searches
}

\author{
Frank J. Tackmann, ${ }^{a}$ Wouter J. Waalewijn ${ }^{b, c}$ and Lisa Zeune ${ }^{c}$ \\ ${ }^{a}$ Theory Group, Deutsches Elektronen-Synchrotron (DESY), \\ D-22607 Hamburg, Germany \\ ${ }^{b}$ ITFA, University of Amsterdam, \\ Science Park 904, 1018 XE, Amsterdam, The Netherlands \\ ${ }^{c}$ Theory Group, Nikhef, \\ Science Park 105, 1098 XG, Amsterdam, The Netherlands \\ E-mail: frank.tackmann@desy.de, wouterw@nikhef.nl, \\ lisa.zeune@nikhef.nl
}

ABSTRACT: Several searches for new physics at the LHC require a fixed number of signal jets, vetoing events with additional jets from QCD radiation. As the probed scale of new physics gets much larger than the jet-veto scale, such jet vetoes strongly impact the QCD perturbative series, causing nontrivial theoretical uncertainties. We consider slepton pair production with 0 signal jets, for which we perform the resummation of jet-veto logarithms and study its impact. Currently, the experimental exclusion limits take the jet-veto cut into account by extrapolating to the inclusive cross section using parton shower Monte Carlos. Our results indicate that the associated theoretical uncertainties can be large, and when taken into account have a sizeable impact already on present exclusion limits. This is improved by performing the resummation to higher order, which allows us to obtain accurate predictions even for high slepton masses. For the interpretation of the experimental results to benefit from improved theory predictions, it would be useful for the experimental analyses to also provide limits on the unfolded visible 0 -jet cross section.

KeYwords: Supersymmetry Phenomenology, Jets

ArXiv EPrint: 1603.03052 


\section{Contents}

1 Overview 1

2 Jet veto resummation $\quad 4$

2.1 Factorization formula 5

2.2 Hard scattering process $\quad 7$

2.3 Estimating the theory uncertainty 9

$\begin{array}{lll}3 & \text { Results } & \mathbf{1 0}\end{array}$

$\begin{array}{lll}3.1 & \text { Slepton production at } 8 \mathrm{TeV} & 10\end{array}$

$\begin{array}{ll}3.2 & \text { Slepton production at } 13 \mathrm{TeV} \\ \end{array}$

4 Conclusions $\quad 15$

$\begin{array}{ll}\text { A Fixed-order ingredients } & 16\end{array}$

A.1 Hard function 16

$\begin{array}{lll}\text { A.2 Beam function } & 17\end{array}$

$\begin{array}{lll}\text { A.3 Soft function } & 18\end{array}$

$\begin{array}{ll}\text { A.4 Nonsingular contributions } & 18\end{array}$

$\begin{array}{lr}\text { B RGE ingredients } & 19\end{array}$

B.1 Anomalous dimensions 20

B.2 Profiles scales 21

\section{Overview}

A crucial challenge at the LHC is to discriminate a faint Beyond-the-Standard Model (BSM) signal from large Standard Model (SM) backgrounds, since for most BSM searches no "smoking gun" signature exists. To eliminate SM backgrounds containing jets, many analyses require a fixed number of hard jets corresponding to the expected number of signal jets in the hard-interaction process. This amounts to placing a veto on additional jets above a certain transverse momentum $p_{T}^{\text {cut }}$ arising from QCD initial-state or final-state radiation. Typical examples are supersymmetry (SUSY) searches for third generation squarks requiring two signal jets and vetoing a third jet [1-3], or electroweakino/slepton searches usually requiring 0 signal jets [4-8]. Jet vetoes are also applied in other BSM searches, including anomalous triple-gauge couplings [9], unparticles [10], large extra dimensions and dark matter candidates in mono-photon, mono- $Z$ and mono-jet events [11-13]. In this paper, we concentrate on slepton (selectron and smuon) searches, focusing in particular on the analysis in ref. [5], which is representative of analyses with no final state jets. Searches 
with jets in the final state are more complicated, as the jet transverse momenta introduce additional kinematic scales in the cross section, and are left for future work.

Exclusion limits require reliable predictions for the expected BSM cross section. So far, the focus of theory calculations has mostly been on the total production cross section, while the effect of exclusive phase-space cuts like jet vetoes has not been much investigated. However, since jet vetoes impose a strong restriction on additional QCD emissions, they can significantly alter the cross section and pose an important source of theory uncertainty, as was observed some time ago in the context of Higgs production [14, 15].

The jet veto introduces large logarithms in the 0-jet cross section, schematically,

$$
\begin{aligned}
\sigma_{0}\left(p_{T}^{\text {cut }}\right)= & a_{00}+\alpha_{s}\left(a_{12} \ln ^{2} \frac{p_{T}^{\text {cut }}}{Q}+a_{11} \ln \frac{p_{T}^{\text {cut }}}{Q}+a_{10}\right) \\
& +\alpha_{s}^{2}\left(a_{24} \ln ^{4} \frac{p_{T}^{\text {cut }}}{Q}+a_{23} \ln ^{3} \frac{p_{T}^{\text {cut }}}{Q}+a_{22} \ln ^{2} \frac{p_{T}^{\text {cut }}}{Q}+a_{21} \ln \frac{p_{T}^{\text {cut }}}{Q}+a_{20}\right) \\
& +\cdots+\left(\text { terms suppressed by } p_{T}^{\text {cut }} / Q\right),
\end{aligned}
$$

where $a_{m n}$ are coefficients and $Q$ denotes the hard-interaction scale, which is set by the (typical) partonic invariant mass, e.g. twice the slepton mass. For $p_{T}^{\text {cut }} \ll Q$, the logarithmic terms produce large corrections leading to a poor perturbative convergence. This can become a large effect for SUSY particle production for which $Q$ can easily be $1 \mathrm{TeV}$ or more, and it will only get more important as the measurements continue to probe higher BSM scales.

The actual experimental limit is on the visible cross section in the fiducial phase space including all experimental reconstruction efficiencies and acceptance cuts, and in particular including the jet veto. Its interpretation in terms of the exclusion limits quoted by the experiments involves the extrapolation from the measured 0-jet cross section to the inclusive cross section using parton shower Monte Carlos. An important outcome of our approach is that we are able to obtain a reliable estimate of the theory uncertainty associated with the jet veto, which parton showers typically do not provide. For this reason, the jet-veto uncertainties, which we find to have a sizeable impact, are also not taken into account in the current results that involve a jet veto.

To obtain accurate theoretical predictions and assess the theoretical uncertainties, the logarithmic terms in eq. (1.1) can be systematically summed up to all orders in $\alpha_{s}$. This resummation for jet vetoes in hadronic collisions has been well-developed in the context of Drell-Yan and Higgs production [14, 16-29], and the same methods have also been used to study diboson processes [30-35].

The $a_{m n}$ coefficients in eq. (1.1) are not all independent, and their structure allows the logarithmic series to be rewritten as

$$
\begin{aligned}
\sigma_{0}\left(p_{T}^{\text {cut }}\right)= & \left(b_{0}+b_{1} \alpha_{s}+\cdots\right) \exp \left[\sum_{m \geq 1}\left(c_{0 m}+c_{1 m} \alpha_{s}+\cdots\right) \alpha_{s}^{m} \ln ^{m+1} \frac{p_{T}^{\text {cut }}}{Q}\right] \\
& +\left(\text { terms suppressed by } p_{T}^{\text {cut }} / Q\right)
\end{aligned}
$$

Each of the series inside round brackets is now free of logarithms, and so can be computed order by order in $\alpha_{s}$. Doing so then amounts to systematically performing the resummation 
to higher logarithmic order. The resummation orders relevant for our discussion include all terms in eq. (1.2) as follows:

$$
\text { LL: } b_{0}, c_{0 m}, \quad \text { NLL: } b_{0}, c_{0 m}, c_{1 m}, \quad \text { NLL': } b_{0}, b_{1}, c_{0 m}, c_{1 m} \text {. }
$$

The $b_{1}$ term, first included at NLL' ${ }^{\prime}$, is important as it incorporates the full one-loop virtual corrections into the resummation, including both QCD and SUSY-QCD corrections. The remaining terms suppressed by $p_{T}^{\text {cut }} / Q$ in eqs. (1.1) and (1.2) start at $\mathcal{O}\left(\alpha_{s}\right)$ and vanish as $p_{T}^{\text {cut }} / Q \rightarrow 0$. At $\mathrm{NLL}^{\prime}+\mathrm{NLO}$ we include them at $\mathcal{O}\left(\alpha_{s}\right)$, which then reproduces the inclusive NLO cross section in the limit $p_{T}^{\text {cut }} \rightarrow \infty$.

We now give a preview of our main results, leaving details of the calculation to section 2 and the appendices. A more extensive discussion with additional plots and results for $\tilde{\ell}_{R} \tilde{\ell}_{R}$ production are given in section 3. Figure 1 shows our resummed predictions for the slepton production cross section with a jet veto at NLL (green band, dotted line) and at NLL'+NLO order (red band, solid line) as a function of the slepton mass $m_{\tilde{\ell}}$ for $8 \mathrm{TeV}$ (left plot) and $13 \mathrm{TeV}$ (right plot). In the left plot we use $p_{T}^{\text {cut }}=20 \mathrm{GeV}$, as in the ATLAS analysis [5], and in the right plot we choose $p_{T}^{\text {cut }}=25 \mathrm{GeV}$ and $100 \mathrm{GeV}$ as representative values. The bands show the perturbative uncertainties (but no parametric PDF uncertainties), which are systematically estimated by varying resummation and renormalization scales, as discussed in detail in section 2.3. The overlap between the bands and the reduction in uncertainties demonstrate the excellent stability of the resummed calculation, allowing us to obtain precise predictions even up to high slepton masses, see right panel, where the impact of the jet veto increases.

To investigate the implications for the exclusion limit, we extract the 95\% CL upper limit on the visible 0-jet cross section from the experimental results by using ATOM [36] and CheckMATE [37] to determine the signal region efficiencies excluding the jet veto. These are shown in the left panel as the dotted and dashed black curves. We translate this into a 95\% CL exclusion limit shown as error bars in the bottom panel, using our NLL prediction (green) or NLL'+NLO prediction (red). This can be compared to the exclusion limit provided by ATLAS (blue) [5], for which the total NLO cross section [38-40] was multiplied with the signal region efficiencies (including the jet veto) obtained using HERWIG++ [41]. (A more consistent combination of the inclusive NLO cross section with parton showers was obtained in ref. [42].) The ATLAS exclusion accounts only for the theory uncertainty associated with the total production cross section, following ref. [43], but does not take into account the uncertainty associated with the jet veto.

The perturbative precision of the parton shower is formally at most that of our NLL results, and hence the perturbative uncertainties due to the jet veto in the experimental limits could easily be as large as that. This has a sizeable impact: using our NLL result the exclusion would go down to $m_{\tilde{\ell}_{L}} \simeq 270 \mathrm{GeV}$. Note that even with our NLL'+NLO predictions the uncertainty on the exclusion is still larger than the one obtained by ATLAS. In the future, it would be advantageous to separate out theory-sensitive acceptance cuts in the experimental results for example by quoting the observed limit on the visible 0 jet cross section with unfolded detector efficiencies. This avoids folding a dominant theory 

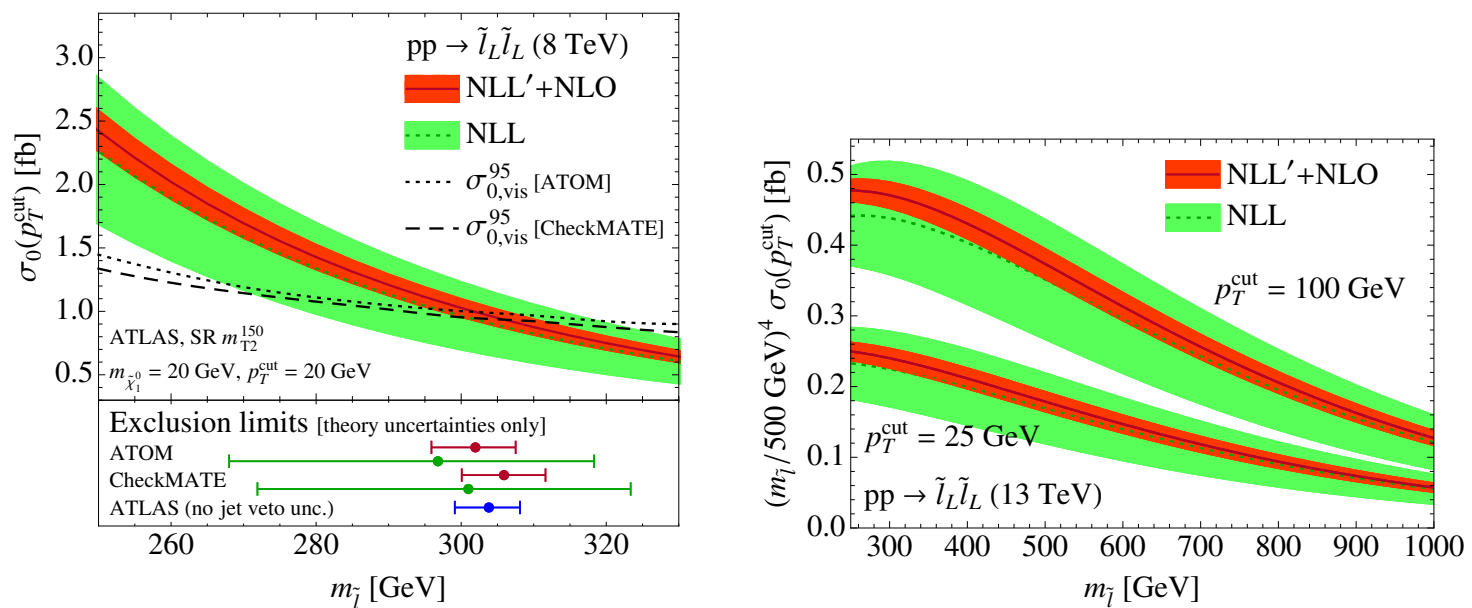

Figure 1. The 0 -jet cross section for $\tilde{\ell}_{L} \tilde{\ell}_{L}$ production as a function of $m_{\tilde{\ell}}$ at $8 \mathrm{TeV}$ (left plot) and $13 \mathrm{TeV}$ (right plot). The results at NLL are shown by the green (light) band and dotted lines and at $\mathrm{NLL}^{\prime}+\mathrm{NLO}$ by the orange (dark) band and solid lines. In the left plot, we use $p_{T}^{\text {cut }}=20 \mathrm{GeV}$ and the dotted and dashed black lines show the experimental 95\% CL upper limit on the visible 0 -jet cross section, which are extracted from the ATLAS results in ref. [5] using ATOM [36] and CheckMATE [37]. The error bars in the bottom panel give the resulting 95\% CL exclusion limits on $m_{\tilde{\ell}}$ using our NLL prediction (green) and $\mathrm{NLL}^{\prime}+\mathrm{NLO}$ prediction (red). This is compared to the 95\% CL exclusion limit provided by ATLAS (blue), which does not take into account the jet-veto uncertainty. In the right plot, we show predictions for the 0 -jet cross section for two representative values of $p_{T}^{\text {cut }}(25 \mathrm{GeV}$ and $100 \mathrm{GeV})$, where the cross section is rescaled by a normalization factor for better visibility.

dependence directly into the quoted exclusion limits and allows the experimental results and their interpretation to easily benefit from future improvements in theoretical predictions.

Finally, we note that soft gluon (threshold) resummation for the total slepton production cross section has been studied extensively in refs. [39, 40, 44, 45]. We emphasize that this type of resummation is separate and can be considered in addition to the jet veto resummation we discuss here. For current values of slepton masses under investigation at the LHC, the effect on the total cross section and uncertainty is rather small, and we therefore do not include it here. The perturbative description with a jet at large $p_{T}$ present in the final state can also be improved by considering the slepton-pair plus jet process [46].

\section{Jet veto resummation}

In this section, we discuss the calculation in some detail. We utilize the jet- $p_{T}$ resummation of ref. [25] using soft-collinear effective theory (SCET) [47-52].

In section 2.1, we present the factorization formula for the process, $p p \rightarrow \tilde{\ell} \tilde{\ell} \rightarrow \ell \chi_{1}^{0} \ell \chi_{1}^{0}$, and discuss how it is used to resum the jet-veto logarithms. Section 2.2 discusses the hard function that describes the underlying short-distance interaction for slepton pair production. In particular, we show that correlations between the jet veto and other kinematic selection cuts are negligible, which will allow us to ignore the slepton decay. In 
section 2.3, we explain how the theoretical uncertainties are estimated through resummation and renormalization scale variations. All fixed-order perturbative ingredients are collected in appendix A, while the anomalous dimensions and scale choices are summarized in appendix B.

\subsection{Factorization formula}

The SCET factorization formula for the 0-jet cross section is given by [19, 20]

$$
\begin{aligned}
\sigma_{0}\left(p_{T}^{\text {cut }}, m_{\mathrm{SUSY}}, \text { cuts }\right)= & \int \mathrm{d} Q^{2} \mathrm{~d} Y H_{q \bar{q}}\left(Q^{2}, Y, m_{\mathrm{SUSY}}, \text { cuts }, \mu\right) \\
& \times B_{q}\left(p_{T}^{\text {cut }}, x_{a}, \mu, \nu\right) B_{\bar{q}}\left(p_{T}^{\text {cut }}, x_{b}, \mu, \nu\right) S_{q \bar{q}}\left(p_{T}^{\text {cut }}, \mu, \nu\right) \\
& +\sigma_{0}^{\text {nons }}\left(p_{T}^{\text {cut }}, m_{\mathrm{SUSY}}, \text { cuts }\right) .
\end{aligned}
$$

Here $Q$ and $Y$ are the total invariant mass and rapidity of the sleptons, and

$$
x_{a}=\frac{Q}{E_{\mathrm{cm}}} e^{Y}, \quad x_{b}=\frac{Q}{E_{\mathrm{cm}}} e^{-Y} .
$$

The hard function $H_{q \bar{q}}$ describes the short-distance scattering process, $q \bar{q} \rightarrow \tilde{\ell} \tilde{\ell} \rightarrow \ell \chi_{1}^{0} \ell \chi_{1}^{0}$. It contains all the analysis cuts applied on the slepton final state but not the jet veto. The relevant SUSY masses are summarized by $m_{\mathrm{SUSY}}$, which in addition to the slepton and neutralino masses also includes the squark and gluino masses at one-loop order (see figure 2(c)). The hard function will be discussed in section 2.2 and appendix A.1.

Due to the jet veto, the real QCD radiation is restricted to be collinear to the beam axis or soft. The beam function $B_{q}\left(B_{\bar{q}}\right)$ describes the effect of the jet veto on collinear initial-state radiation from the colliding (anti)quark with momentum fraction $x_{a}\left(x_{b}\right)$, and combines the nonperturbative parton distribution functions (PDFs) with perturbative initial-state radiation [16]. The restriction of the jet veto on soft radiation is encoded in the soft function $S_{q \bar{q}}$. The required NLO results for the beam and soft functions are given in appendix A.2 and appendix A.3. The dependence on the jet algorithm and jet radius effects first appear at NNLL in $\ln \left(p_{T}^{\text {cut }} / Q\right)$ and $\mathcal{O}\left(\alpha_{s}^{2}\right)[19-21]$ and are beyond the order we consider here.

The nonsingular cross section $\sigma^{\text {nons }}$ in eq. (2.1) only consists of the $\mathcal{O}\left(p_{T}^{\text {cut }} / Q\right)$ suppressed terms already mentioned in eq. (1.2) and vanishes for $p_{T}^{\text {cut }} \rightarrow 0$. In appendix A.4 we describe how the nonsingular terms are obtained.

Eq. (2.1) factorizes the large jet veto logarithms. For example, the leading double logarithm in the NLO cross section splits up as

$$
\ln ^{2} \frac{p_{T}^{\text {cut }}}{Q}=\ln ^{2} \frac{Q}{\mu}+2 \ln \frac{p_{T}^{\text {cut }}}{\mu} \ln \frac{\nu}{Q}+\ln \frac{p_{T}^{\text {cut }}}{\mu} \ln \frac{\mu p_{T}^{\text {cut }}}{\nu^{2}},
$$

where the three terms on the right-hand side are the contributions from the NLO hard, beam, and soft functions, respectively. The key to obtaining a resummed prediction for the cross section is that each individual term can be made small by an appropriate choice of the renormalization scale $\mu$ and rapidity renormalization scale $\nu$, namely

$$
\mu_{H} \sim Q \sim 2 m_{\tilde{\ell}}, \quad \mu_{B} \sim \mu_{S} \sim p_{T}^{\text {cut }}, \quad \nu_{B} \sim Q \sim 2 m_{\tilde{\ell}}, \quad \nu_{S} \sim p_{T}^{\text {cut }} .
$$




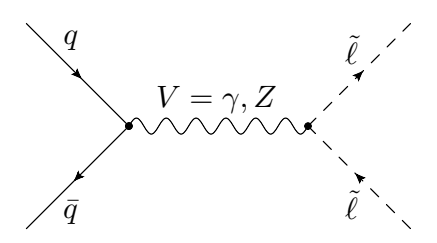

(a) Leading order
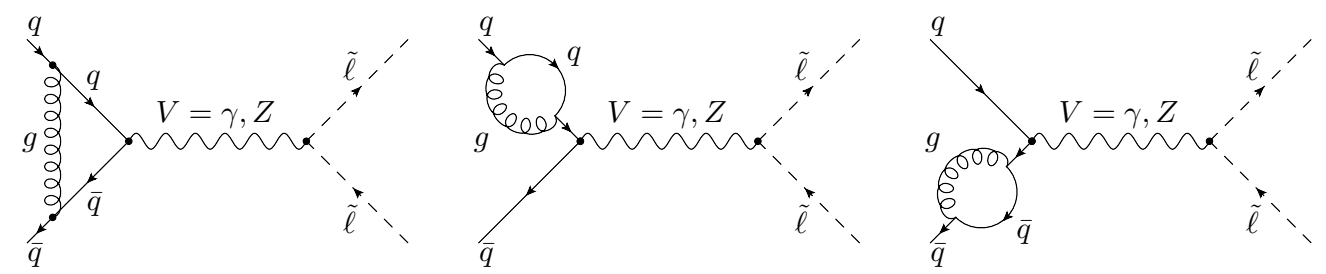

(b) One-loop QCD corrections
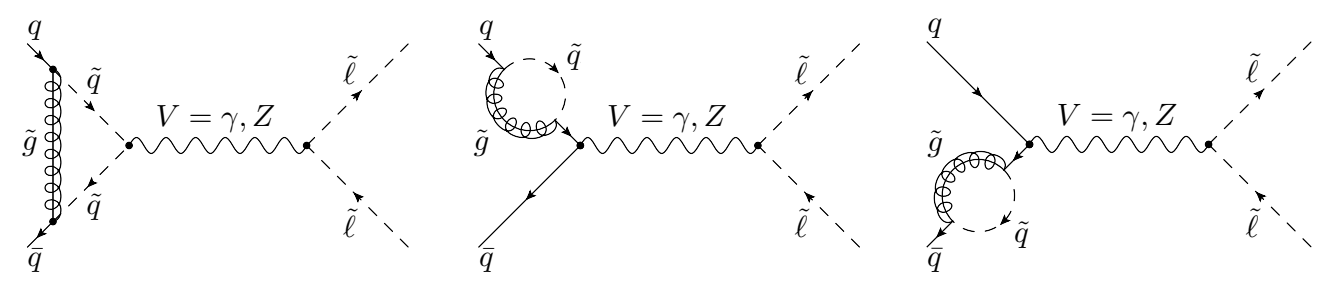

(c) One-loop SUSY-QCD corrections

Figure 2. Leading order and one-loop virtual corrections to slepton pair production.

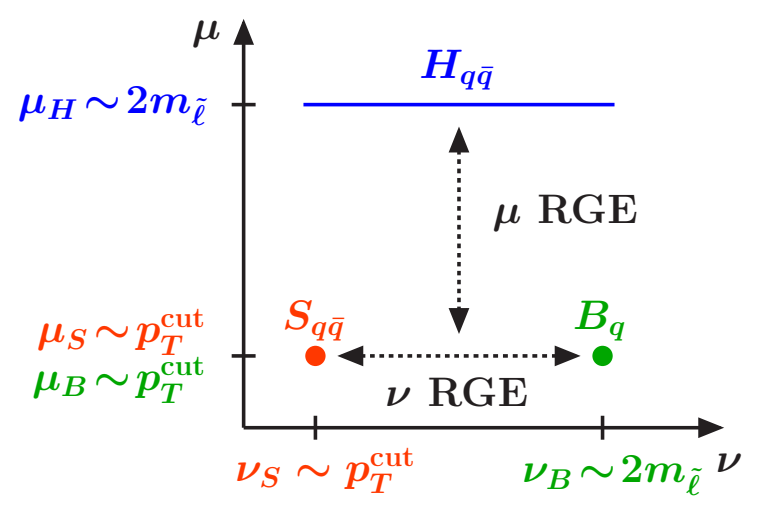

Figure 3. The hard, beam, and soft functions are evolved in virtuality $\mu$ from their natural scales $\mu_{H} \sim 2 m_{\tilde{\ell}}$ and $\mu_{B} \sim \mu_{S} \sim p_{T}^{\text {cut }}$. The beam and soft functions are also evolved in rapidity $\nu$ from their natural scales $\nu_{B} \sim 2 m_{\tilde{\ell}}$ and $\nu_{S} \sim p_{T}^{\text {cut }}$.

By evaluating each of the hard, beam, and soft functions at their natural scale, they contain no large logarithms. The logarithms in the cross section are then efficiently resummed by evolving each of the functions using their renormalization group evolution (RGE) for $\mu$ and the rapidity RGE for $\nu[53,54]$ to the common (and arbitrary) scales $\mu$ and $\nu$ at which the cross section in eq. (2.1) is evaluated. The RGE is illustrated in figure 3 and the formulae needed for carrying it out are collected in appendix B. 


\section{$2.2 \quad$ Hard scattering process}

We now discuss the hard function, which contains the hard scattering process $q \bar{q} \rightarrow \tilde{\ell} \tilde{\ell} \rightarrow$ $\ell \chi_{1}^{0} \ell \chi_{1}^{0}$ including the tree-level and virtual loop corrections shown in figure 2 . We consider a simplified (R-parity conserving) model where all SUSY particles except for the slepton $\tilde{\ell}$ and the lightest neutralino $\chi_{1}^{0}$ are heavy and $\mathcal{B}\left(\tilde{\ell} \rightarrow \ell \chi_{1}^{0}\right)=1$. We will argue that we can simply calculate inclusive slepton production with a jet veto, without considering the subsequent decay of the sleptons, since the jet veto is uncorrelated with the other cuts on the slepton decay products. The resulting hard function is given in appendix A.1.

The jet veto is factorized from the other cuts in eq. (2.1), since only the soft and beam functions depend on the jet veto, whereas the hard function depends on the other cuts. ${ }^{1}$ Hence, the only possibility to introduce correlations between the jet veto and other cuts is through the common variables $Q^{2}$ and $Y{ }^{2}$ If the cuts were to induce sizeable changes in the $Q^{2}$ and $Y$ dependence of the hard function, then the $p_{T}^{\text {cut }}$-dependent beam and soft functions would get weighted in a cut-dependent way when integrated over $Q^{2}$ and $Y$.

We have investigated this using MadGraph (version 2.3.2) [56] for the signal regions SR- $m_{T 2}$ of ref. [5], which consist (besides the jet veto) of the following cuts:

- Two (same-flavor) leptons with $p_{T}>35 \mathrm{GeV}$ and $p_{T}>20 \mathrm{GeV}$. The pseudorapidity of each lepton is required to be $|\eta|<2.47$ for electrons and $|\eta|<2.4$ for muons.

- The dilepton invariant mass $m_{\ell \ell}>20 \mathrm{GeV}$ and $\left|m_{\ell \ell}-m_{Z}\right|>10 \mathrm{GeV}$.

- Three possible cuts on the stransverse mass $[57,58] m_{T 2}>90,120$, or $150 \mathrm{GeV}$.

The resulting tree-level cross section, corresponding to the tree-level hard function, is shown in figure 4 for a selectron mass of $250 \mathrm{GeV}$ and a neutralino mass of $20 \mathrm{GeV}$. The gray line shows the number of events per bin without cuts and the colored lines show the number of events after the signal region cuts. The bands indicate the statistical uncertainty due to the number of simulated events. The top and bottom rows show the $Q$ and $Y$ dependence, respectively. In the right column, each bin is normalized to the total number of events in that bin, i.e., showing the acceptance of the cuts in each $Q$ and $Y$ bin. We can see that the cut acceptance is essentially flat in $Q$ and $Y$, so these cuts do not affect the shape in $Q$ and $Y$ but only the normalization. The $Y$ dependence is no longer flat for $|Y|>1.5$, but this corresponds to only $8 \%$ of the total cross section. This implies that to very good approximation we can treat the other cuts as a $Q$ and $Y$ independent multiplicative correction which we can factor out from eq. (2.1). This treatment is completely sufficient for our purposes, since in order to compare to the experimental measurements we will also have to include experimental reconstruction efficiencies, which we are anyway only able to

\footnotetext{
${ }^{1}$ The kinematic cuts considered here affect only the hard kinematics. If one would have additional IRsensitive cuts that resolve the hadronic final state, the factorization in eq. (2.1) would get more complicated, see e.g. ref. [55] for a prototypical situation.

${ }^{2}$ In principle, the hard function is independent of the boost $Y$, however the cuts are not. In addition, the nonsingular corrections $\sigma_{0}^{\text {nons }}$ depend on both the jet veto and the other cuts, but these corrections are negligible in the relevant region of $p_{T}^{\text {cut }} \ll Q$.
} 

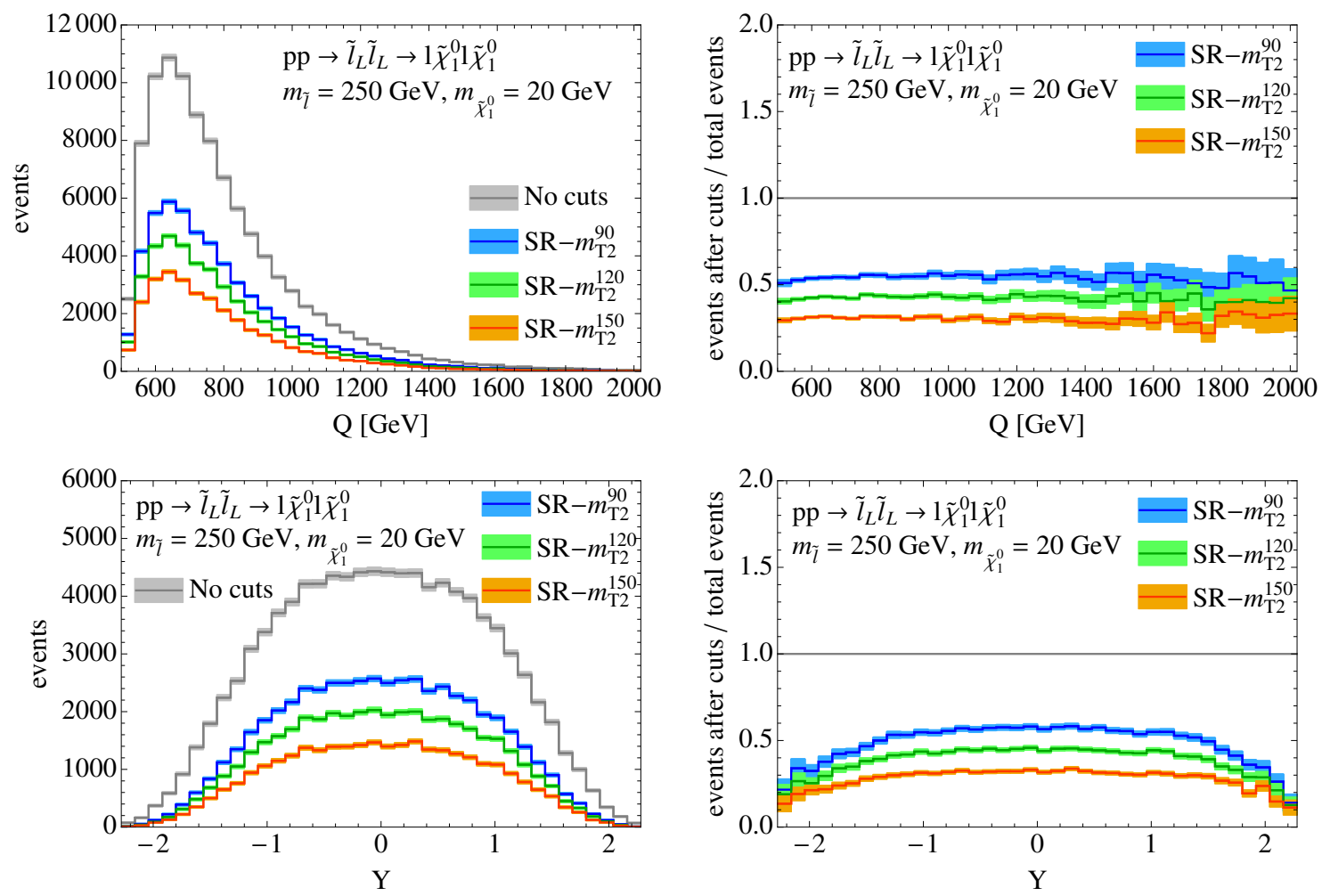

Figure 4. The effect of the signal region cuts (besides the jet veto) on the $Q$ (upper row) and $Y$ (lower row) dependence of the cross section. The left column shows the number of events per bin, before (gray) and after (blue, green and orange) cuts. The right column shows the acceptance per bin.

do approximately. Hence, we focus our attention on the jet-veto cut, which receives large QCD corrections, without considering the other cuts.

Once we restrict ourselves to only calculating the jet veto, the assumption that $\mathcal{B}(\tilde{\ell} \rightarrow$ $\left.\ell \chi_{1}^{0}\right)=1$ allows us to focus on slepton production without the subsequent decay. We do not consider mixing in the slepton sector and we separately discuss $\tilde{\ell}_{L} \tilde{\ell}_{L}$ and $\tilde{\ell}_{R} \tilde{\ell}_{R}$ production. ${ }^{3}$ This is a good approximation for sleptons of the first two generations, which we focus on here. For staus, mixing effects are relevant and can be easily included.

At tree level, slepton pairs are produced via a $q \bar{q}$-initiated $s$-channel exchange of a photon $\gamma$ or a $Z$ boson, as shown in figure 2(a). The leading-order hard function is simply equal to the corresponding partonic cross section, which has been calculated in refs. [59-62]. Since the intermediate $\gamma / Z$ decays into a noncolored final state, the one-loop QCD corrections affect only the $q \bar{q} V$ production vertex and are identical to those of the Drell-Yan process [63], see figure 2(b). The one-loop SUSY-QCD corrections are shown in figure 2(c). They have been calculated in ref. [38] neglecting squark mixing and in ref. [64] including squark mixing. In the simplified model considered here, the squarks are heavy and SUSY-

\footnotetext{
${ }^{3} \tilde{\ell}_{L}\left(\tilde{\ell}_{R}\right)$ denotes the superpartner of a left-handed (right-handed) lepton $\ell$ and will be referred to as a left-handed (right-handed) slepton.
} 
QCD corrections are small compared to the QCD corrections. Mixing effects in the squark sector are therefore neglected. The resulting NLO hard function is given in appendix A.1. If squark mixing effects become relevant, they can be straightforwardly included in the hard function. Note also that at one-loop order gluon-initiated slepton production is in principle also possible via a Higgs or quartic scalar coupling $[65,66]$. However, the corresponding cross section is very small (except in the resonance region) and is therefore not considered here (or in Prospino).

\subsection{Estimating the theory uncertainty}

In this section, we discuss the resummation scales that are used to obtain the central value for the cross section and to assess the perturbative uncertainty, with additional details relegated to appendix B.2. We have also evaluated the parametric PDF uncertainty for the resummed 0-jet cross section, which is explained in the discussion of figure 10 in section 3 below.

In SCET, resummation is performed by evaluating the hard, beam, and soft functions at their natural virtuality and rapidity resummation scales and then evolving them to common $\mu$ and $\nu$ scales using their virtuality and rapidity RG equations, as illustrated in figure 3. The resummation is crucial for $p_{T}^{\text {cut }} \ll Q \sim 2 m_{\tilde{\ell}}$, but must be switched off for large $p_{T}^{\text {cut }}$ to correctly reproduce the fixed-order cross section in that region. The smooth transition between the resummation and fixed-order regions is achieved by using $p_{T}^{\text {cut }}$ depended resummation scales, called profile scales. Profile scales were first introduced to study the $B \rightarrow X_{s} \gamma$ spectrum [67] and the thrust event shape in $e^{+} e^{-}$collisions [68]. They have since been applied in many resummed calculations and a variety of different contexts (see e.g. refs. [14, 23, 25, 67-78]) and are established as a reliable method to assess the perturbative uncertainty in resummed predictions. Our profile scales are constructed by considering the relative size of the singular and nonsingular cross section contributions, as discussed in appendix B.2. They are shown in figure 5, where solid curves correspond to the central scale choice and dotted curves correspond to variations that are used to estimate the perturbative uncertainty, as discussed below.

Our procedure for estimating the perturbative uncertainty using profile scale variations follows ref. [25]. The perturbative uncertainty $\Delta_{0}$ on the 0 -jet cross section is given by

$$
\Delta_{0}^{2}=\left(\Delta_{\mu 0}\right)^{2}+\Delta_{\text {resum }}^{2}
$$

where $\Delta_{\mu 0}$ reproduces the standard fixed-order uncertainties in the limit of large $p_{T}^{\text {cut }}$, whereas the resummation uncertainty $\Delta_{\text {resum }}$ associated with the jet veto vanishes in the large $p_{T}^{\text {cut }}$ region. Both $\Delta_{\mu 0}$ and $\Delta_{\text {resum }}$ are estimated via profile scale variations, shown in figure 5 .

The set of profile variations $V_{\mu}$ contributing to $\Delta_{\mu 0}$ are displayed in the left panel of figure 5. They vary the overall scale by a factor $1 / 2$ and 2 as well as the parameters that control the transition points between resummation and fixed-order regions. For each profile $v_{i}$ in $V_{\mu}$ we calculate the 0 -jet cross section $\sigma_{0}^{v_{i}}$, from which we obtain $\Delta_{\mu 0}$ by taking the 

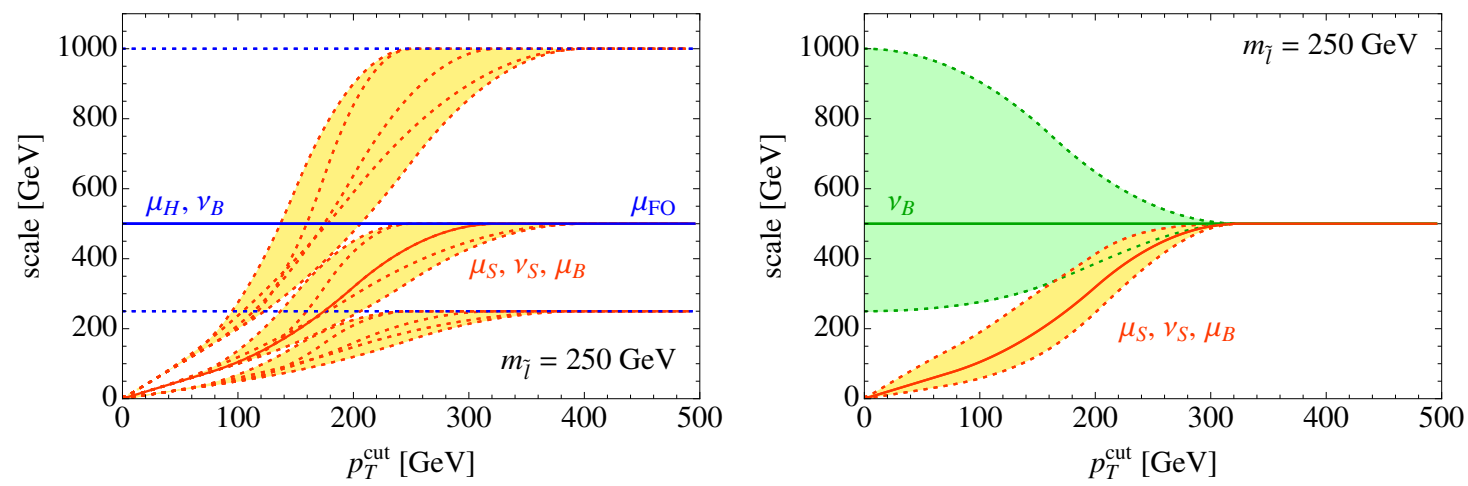

Figure 5. Profile functions and their variations used to determine the theory uncertainty, as explained in the text. Left: profile functions for $\mu_{H}, \nu_{B}$ in blue and for $\mu_{B}, \mu_{S}, \nu_{S}$ in red. Solid lines show the central scale choice, while dotted lines show the variations contributing to $\Delta_{\mu 0}$ where the yellow shading is between the profiles belonging to the same value of $\mu_{\mathrm{FO}}$. Right: variations of $\nu_{B}$ (green lines and shading) and $\mu_{B}, \mu_{S}, \nu_{S}$ (red lines and yellow shading) contributing to $\Delta_{\text {resum }}$.

(symmetrized) envelope,

$$
\Delta_{\mu 0}\left(p_{T}^{\text {cut }}\right)=\max _{v_{i} \in V_{\mu}}\left|\sigma_{0}^{v_{i}}\left(p_{T}^{\text {cut }}\right)-\sigma_{0}^{\text {central }}\left(p_{T}^{\text {cut }}\right)\right| .
$$

The profile scale variations $V_{\text {resum }}$ contributing to $\Delta_{\text {resum }}$ are shown in the right panel of figure 5 . They separately vary each of the beam and soft $\mu$ and $\nu$ scales up and down but keep the hard scale $\mu_{H}=\mu_{\mathrm{FO}}$ fixed. They thus directly probe the size of the logarithms and the associated resummation uncertainty, while smoothly turning off as the resummation itself is turned off. This yields the following estimate for $\Delta_{\text {resum }}$,

$$
\Delta_{\text {resum }}\left(p_{T}^{\text {cut }}\right)=\max _{v_{i} \in V_{\text {resum }}}\left|\sigma_{0}^{v_{i}}\left(p_{T}^{\text {cut }}\right)-\sigma_{0}^{\text {central }}\left(p_{T}^{\text {cut }}\right)\right| .
$$

For additional details on the profile variations we refer to appendix B.2 and ref. [25].

\section{Results}

In this section, we discuss our results for the 0 -jet cross section, $\sigma_{0}$, for slepton production at 8 and $13 \mathrm{TeV}$ and discuss the implications on current slepton exclusion limits, using the ATLAS analysis in ref. [5] as a representative example.

\subsection{Slepton production at $8 \mathrm{TeV}$}

We start by presenting our $8 \mathrm{TeV}$ results. In figure 6 , we show the $p_{T}^{\text {cut }}$ dependence of the 0 -jet cross section. This allows us to discuss the transition between the resummation and fixed-order regions, as well as the perturbative convergence and uncertainties. We consider the implications for the ATLAS exclusion limit in figure 7. The CTEQ6L1 PDFs [79] are used for the plots in this section, to remain consistent with the ATLAS analysis [5]. We show separate results for the direct production of left-handed and right-handed sleptons, focusing on the edge of the $8 \mathrm{TeV}$ exclusion limits $[5,6]$. 

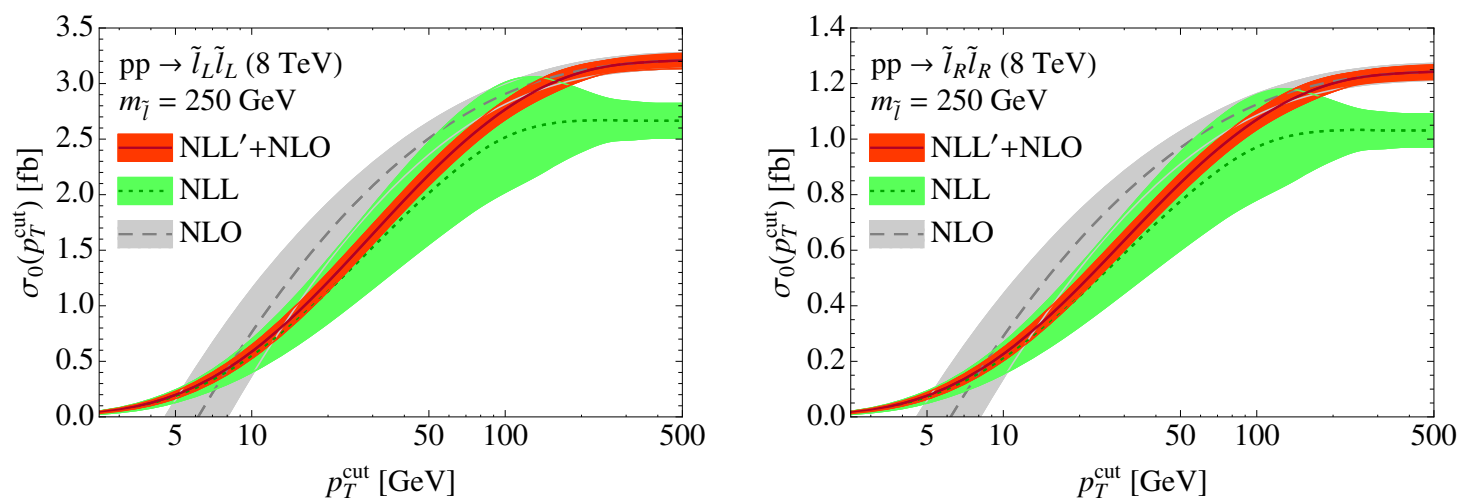

Figure 6. The 0 -jet cross section for $\tilde{\ell}_{L} \tilde{\ell}_{L}$ (left) and $\tilde{\ell}_{R} \tilde{\ell}_{R}$ (right) production at $8 \mathrm{TeV}$ as a function of the jet veto, $p_{T}^{\text {cut }}$. We compare the results at NLL (green band, dotted line), NLL'+NLO (orange band, solid line), and fixed NLO (gray, dashed line), where the bands show the respective perturbative uncertainties.
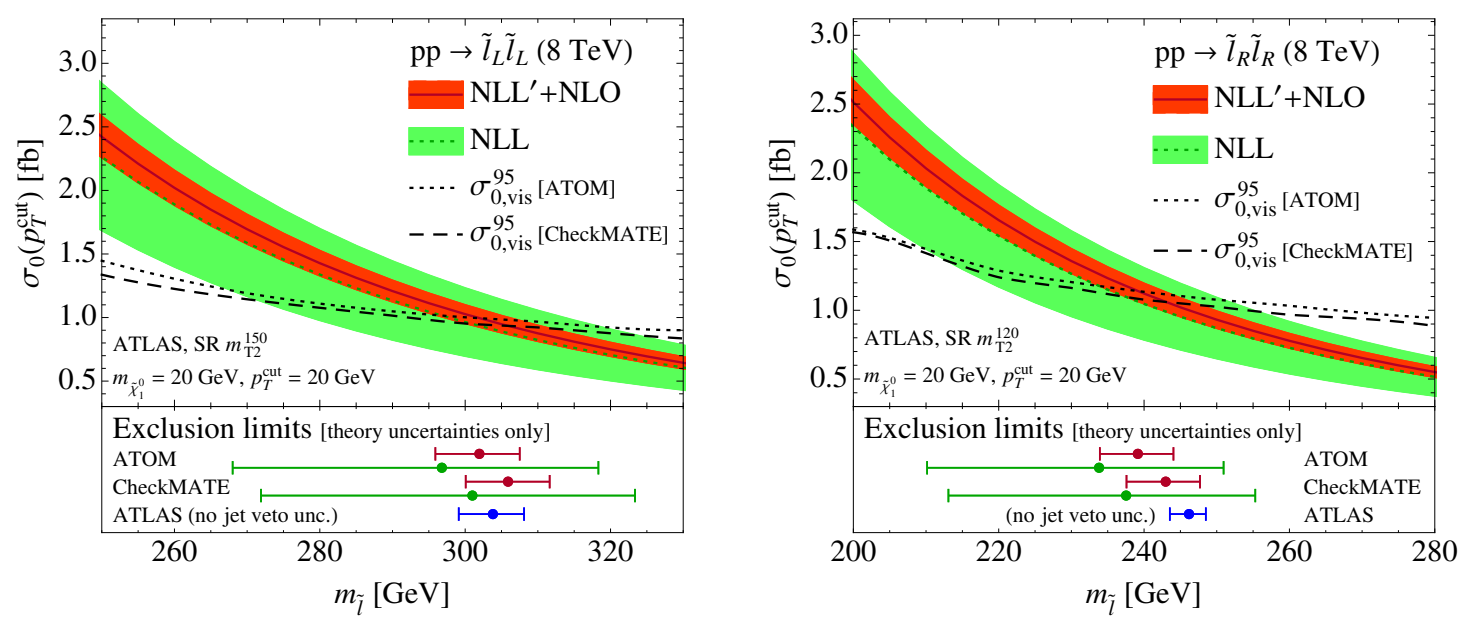

Figure 7. The 0-jet cross section for $\tilde{\ell}_{L} \tilde{\ell}_{L}$ (left) and $\tilde{\ell}_{R} \tilde{\ell}_{R}$ (right) production as a function of $m_{\tilde{\ell}}$ at $8 \mathrm{TeV}$. Shown are our NLL (green band, dotted line) and NLL'+NLO (red band, solid line) predictions, as well as the observed 95\% CL upper limit on the visible 0-jet cross section, using ATOM (black dotted line) and CheckMATE (black dashed line) to determine the signal region efficiencies. The error bars in the lower panels show the 95\% CL exclusion limits obtained from our NLL prediction (green) and $\mathrm{NLL}^{\prime}+\mathrm{NLO}$ prediction (red), and for comparison the limit provided by ATLAS (blue).

Our predictions for the 0 -jet cross section at $8 \mathrm{TeV}$ are shown in figure 6 as a function of $p_{T}^{\text {cut }}$ for $\tilde{\ell}_{L} \tilde{\ell}_{L}$ (left panel) and $\tilde{\ell}_{R} \tilde{\ell}_{R}$ (right panel) production. We take $m_{\tilde{\ell}}=250 \mathrm{GeV}$ as a representative value, ${ }^{4}$ and treat other SUSY particles as decoupled. The predictions are shown at NLO (gray band, dashed line), NLL (green band, dotted line) and NLL'+NLO (red band, solid line). The scale choice for the central value (line) and method for estimating

\footnotetext{
${ }^{4}$ For right-handed selectrons or smuons the exclusion limits are $\sim 200 \mathrm{GeV}$, whereas for left-handed sleptons they are $\sim 275 \mathrm{GeV}$.
} 
the perturbative uncertainty (band) were discussed in section 2.3 and appendix B.2 for the resummed predictions. For the NLO prediction, we use the fixed-order scale $\mu_{\mathrm{FO}}=$ $2 m_{\tilde{\ell}}=500 \mathrm{GeV}$ for the central value and estimate the perturbative uncertainty with the ST method [15]. The latter avoids that the naive fixed-order scale variations typically underestimate the perturbative uncertainty in the fixed-order predictions for small $p_{T}^{\text {cut }}$ due to cancellations between perturbative corrections to the total cross section and those related to the jet veto.

In the region $p_{T}^{\text {cut }} \ll Q$, the large logarithms spoil the applicability of the fixed-order perturbative expansion and eventually drive the NLO cross section negative. A jet veto of $20 \mathrm{GeV}$, as used in the ATLAS analysis [5], sits deep inside this resummation region. We observe that our best prediction at $\mathrm{NLL}^{\prime}+\mathrm{NLO}$ is significantly lower than the fixed NLO result. On the other hand, fixed-order perturbation theory does provide a reliable prediction at large values of $p_{T}^{\text {cut }}$, where the resummation must be turned off. Accordingly, the NLL'+NLO prediction smoothly merges into the NLO result, for which the nonsingular contribution to the cross section is important, as discussed in appendix A.4. We have verified that in the limit of large $p_{T}^{\text {cut }}$ our $\mathrm{NLL}^{\prime}+\mathrm{NLO}$ prediction exactly reproduces the NLO total cross section of Prospino. ${ }^{5}$ Comparing the NLL and NLL'+NLO uncertainty bands, we find that the increased resummation and matching order leads to a substantial reduction of the uncertainties with the NLL'+NLO band fully inside the NLL uncertainty band (except in the fixed-order region where the uncertainties match those of the fixedorder total cross section).

Next, we investigate the implications of our resummed 0-jet slepton production cross section for the ATLAS exclusion limit [5]. In their results, the visible cross section in signal region $a$ is calculated as

$$
\sigma_{\text {vis }}=\sigma(p p \rightarrow \tilde{\ell} \tilde{\ell}) \times \epsilon^{(a)},
$$

where $\epsilon^{(a)}$ contains both the reconstruction efficiencies and the acceptance for the cuts of signal region $a$. They use the total cross section $\sigma(p p \rightarrow \tilde{\ell} \tilde{\ell})$ at NLO from Prospino2.1 [38], checked against Resummino [39, 40], and determine $\epsilon^{(a)}$ using events generated by HERWIG++ v2.5.2 [41] using the CTEQ6L1 PDF set. The resulting $\sigma_{\text {vis }}$ is then compared to the measured $95 \%$ CL upper limit on the visible BSM cross section $\sigma_{\text {vis }}^{95}$ in the signal region $a$.

To compare the $\sigma_{\text {vis }}^{95}$ reported by ATLAS to our predictions, we determine the upper limit on the visible 0 -jet cross section as

$$
\sigma_{0, \mathrm{vis}}^{95}=\frac{\sigma_{\mathrm{vis}}^{95}}{\epsilon^{(a-\mathrm{no} \mathrm{JV})}}, \quad \epsilon^{(a)}=\epsilon^{(a-\mathrm{no} \mathrm{JV})} \epsilon^{\mathrm{JV}} .
$$

Here, $\epsilon^{(a-n o J V)}$ is the signal region efficiency including reconstruction efficiencies and acceptance cuts but excluding the jet veto cut. In other words, we separate the total signal region efficiency $\epsilon^{(a)}$ into the product of $\left.\epsilon^{(a-n o J V}\right)$ and the jet veto efficiency $\epsilon^{\mathrm{JV}}$. Excluding the latter effectively avoids having to rely on the Monte Carlo to correctly describe the effect of the jet veto. The resulting $\sigma_{0 \text {,vis }}^{95}$ is now defined without reconstruction efficiencies and without acceptance cuts other than the jet veto. To model the ATLAS analysis and

\footnotetext{
${ }^{5}$ The default value for the fixed-order scale in Prospino is $m_{\tilde{\ell}}$, which we changed to $2 m_{\tilde{\ell}}$ for this comparison.
} 
determine the signal region efficiencies, we employ ATOM [36] and CheckMATE [37]. ${ }^{6}$ Using the cut-flow tables provided by ATLAS for $m_{\tilde{\ell}}=250 \mathrm{GeV}$ and $m_{\tilde{\chi}_{1}^{0}}=10 \mathrm{GeV}$, we validated both the ATOM and CheckMATE results for $\epsilon^{(a-\text { noJV })}$ for the signal regions $a=m_{T 2}^{120}$ and $m_{T 2}^{150}$ of ref. [5] and found agreement at the 5-10\% level.

Figure 7 shows the results for $\sigma_{0 \text {,vis }}^{95}$ as a function of the slepton mass (for a neutralino with $m_{\tilde{\chi}_{1}^{0}}=20 \mathrm{GeV}$ ), obtained with ATOM (dotted black line) and CheckMATE (dashed black line). This can be directly compared to our resummed predictions for 0-jet slepton production at NLL (green band, dotted line) and at NLL'+NLO (red band, solid line). Note that we show here the combined cross section for mass degenerate selectrons and smuons, whereas all other plots (except the left panel of figure 1) are for one generation of sleptons.

The ATLAS exclusion limits were determined using the signal region with the highest expected sensitivity, which is $m_{T 2}^{150}\left(m_{T 2}^{120}\right)$ near the exclusion for left-handed (right-handed) sleptons around $m_{\tilde{\ell}_{L}} \sim 300 \mathrm{GeV}\left(m_{\tilde{\ell}_{R}} \sim 250 \mathrm{GeV}\right)$. We chose these signal regions in figure 7 , neglecting the possibility that the signal region with the highest expected sensitivity might change within the plotted range. The intersections of the $\sigma_{0, \text { vis }}^{95}$ curves with our resummed predictions set our NLL and $\mathrm{NLL}^{\prime}+\mathrm{NLO}$ exclusion limits, ${ }^{7}$ shown by the green and red error bars in the lower panels of the plots. The blue error bars in the lower panels show for comparison the current exclusion limits as quoted by ATLAS, which account for the theory uncertainty on the total cross section (including PDF uncertainties) following ref. [43]. However, this does not include the uncertainty induced by the jet veto, which could easily be as large as our NLL uncertainty, since the perturbative precision of parton showers to model the jet veto is at best NLL. ${ }^{8}$ At NLL the exclusion limits are noticeably weaker and would go down to $\sim 270 \mathrm{GeV}$ for left-handed sleptons and $\sim 210 \mathrm{GeV}$ for righthanded sleptons. Even our NLL' $+\mathrm{NLO}$ results (without including $\mathrm{PDF}+\alpha_{s}$ uncertainties) yield somewhat larger uncertainties. Encouragingly, the overall central values of our best exclusion limits are similar to those obtained by ATLAS. They agree well in the left panel $\left(\tilde{\ell}_{L} \tilde{\ell}_{L}\right)$ and are slightly lower in the right plot $\left(\tilde{\ell}_{R} \tilde{\ell}_{R}\right)$. However, the overall central values should be treated with some caution as they rely on the signal efficiencies from ATOM and CheckMATE, which have 5-10\% uncertainties. To draw any firm conclusions on the final limits, the experimental analyses would need to provide results for $\sigma_{0 \text {,vis }}^{95}$ or to directly implement our improved theoretical predictions and uncertainties in their interpretations.

\subsection{Slepton production at $13 \mathrm{TeV}$}

We continue our discussion with the 0 -jet cross section for slepton production at $13 \mathrm{TeV}$. Following the PDF4LHC recommendations [86], we use the PDF4LHC15_nlo_mc PDF set in this section. In figure 8 , we show our resummed results for the 0 -jet cross section as a

\footnotetext{
${ }^{6}$ Both programs use FastJet [80] and utilize the $m_{T 2}$ variable [57, 58, 81, 82]. CheckMATE applies Delphes 3 [83] for detector simulation, whereas ATOM builds on RIVET [84]. A detailed description and validation of ATOM can be found in ref. [85].

${ }^{7}$ We simply exclude the regions where the calculated 0 -jet cross section is larger than the upper limit, $\sigma_{0, \text { vis }}^{95}$, without calculating a CLs value.

${ }^{8}$ Note that the Monte Carlo predictions are reweighted to the total NLO cross section. This is equivalent to rescaling the NLL green band in figure 6 to match the NLO result at large $p_{T}^{\text {cut }} \gtrsim 2 m_{\tilde{\ell}}$ and does not improve the resummation precision.
} 

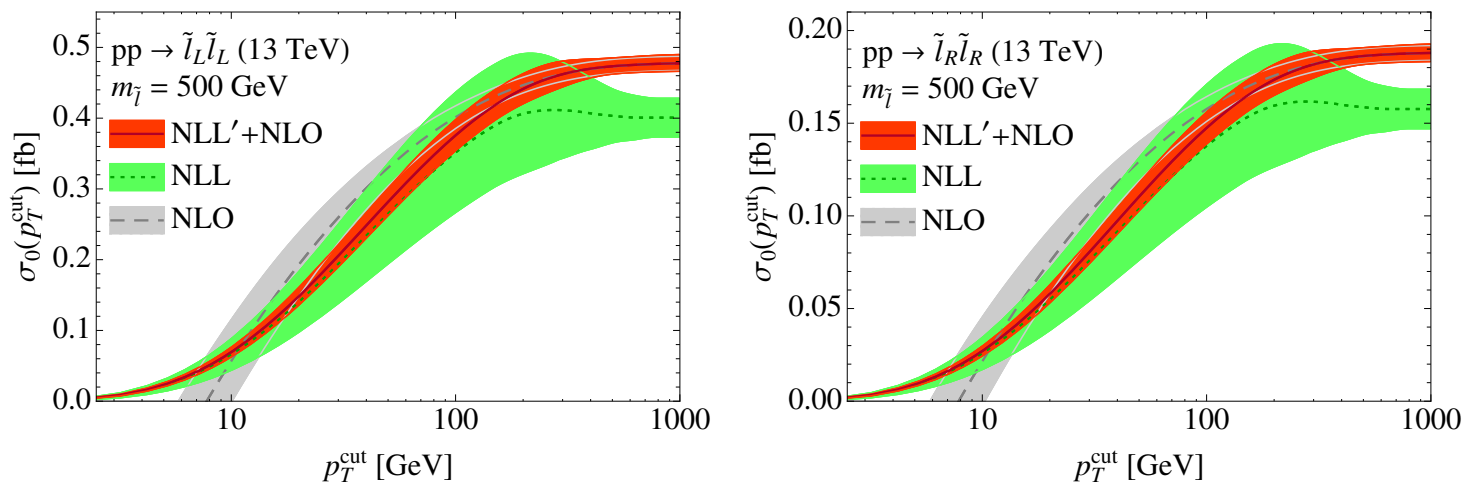

Figure 8. The 0-jet cross section for $\tilde{\ell}_{L} \tilde{\ell}_{L}$ (left) and $\tilde{\ell}_{R} \tilde{\ell}_{R}$ (right) production as a function of $p_{T}^{\text {cut }}$ for $m_{\tilde{\ell}}=500 \mathrm{GeV}$ at $13 \mathrm{TeV}$. The bands show the perturbative uncertainties.
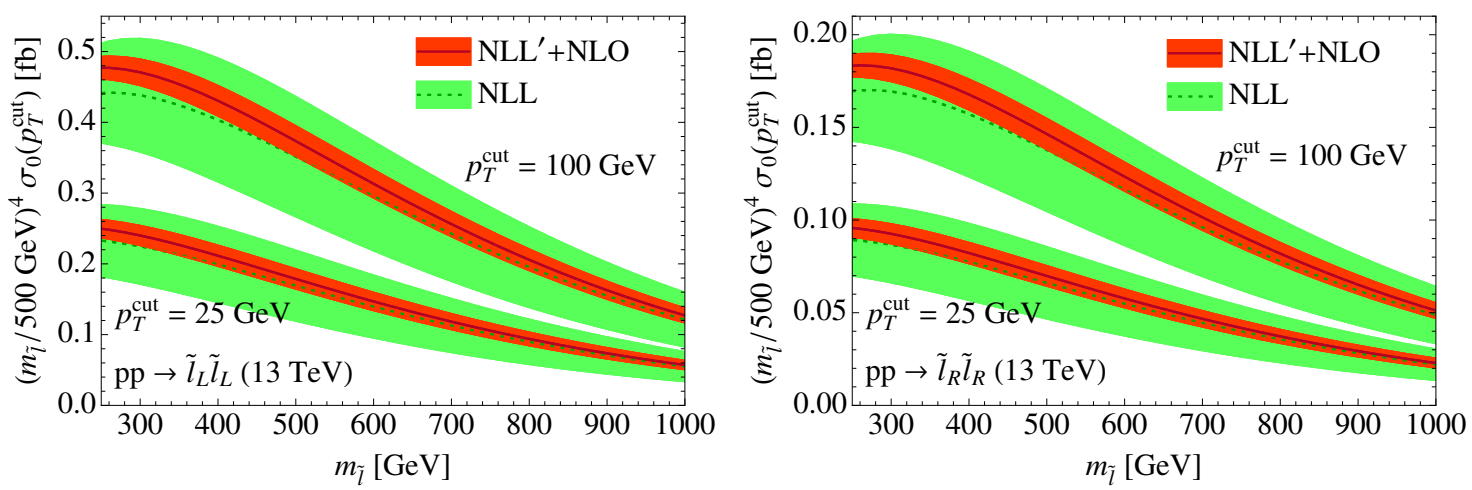

Figure 9. The 0 -jet cross section for $\tilde{\ell}_{L} \tilde{\ell}_{L}$ (left) and $\tilde{\ell}_{R} \tilde{\ell}_{R}$ (right) production as a function of $m_{\tilde{\ell}}$ for $p_{T}^{\text {cut }}=25 \mathrm{GeV}$ and $100 \mathrm{GeV}$ at $13 \mathrm{TeV}$. Shown are the NLL (green band, dotted line) and $\mathrm{NLL}^{\prime}+\mathrm{NLO}$ (red band, solid line) predictions with their perturbative uncertainty. We multiply the cross section by $\left(m_{\tilde{\ell}} / 500 \mathrm{GeV}\right)^{4}$ for better visibility.

function of $p_{T}^{\text {cut }}$ for $m_{\tilde{\ell}}=500 \mathrm{GeV}$. Comparing this to the $8 \mathrm{TeV}$ results with $m_{\tilde{\ell}}=250 \mathrm{GeV}$ in figure 6 , we observe an increase in the perturbative uncertainties. This is expected due to the higher slepton mass, which leads to larger logarithms in the cross section.

In figure 9, we show the resummed 0-jet cross section as a function of $m_{\tilde{\ell}}$ for $p_{T}^{\text {cut }}=$ $25 \mathrm{GeV}$ and $100 \mathrm{GeV}$. The nonsingular contribution is small enough that we can neglect it in this plot. ${ }^{9}$ The overlap between the NLL and NLL'+NLO bands illustrates again the excellent stability of our resummed calculation.

In figure 10 we focus on the uncertainties, normalizing all results to the central NLL'+NLO result. The 0-jet cross section for left-handed slepton production is shown for $p_{T}^{\text {cut }}=25 \mathrm{GeV}$ (left panel) and $p_{T}^{\text {cut }}=100 \mathrm{GeV}$ (right panel) at NLL (green band, dotted lines) and NLL'+NLO (red band, solid lines). Furthermore, the yellow band shows the PDF uncertainty of the NLL'+NLO result, obtained using the standard deviation approach

\footnotetext{
${ }^{9}$ Even for $p_{T}^{\text {cut }}=100 \mathrm{GeV}$ and $m_{\tilde{\ell}}=250 \mathrm{GeV}$ where it is least suppressed the nonsingular correction is only $\sim 1 \%$. For larger $m_{\tilde{\ell}}$ and smaller $p_{T}^{\text {cut }}$ the nonsingular contribution is significantly smaller.
} 

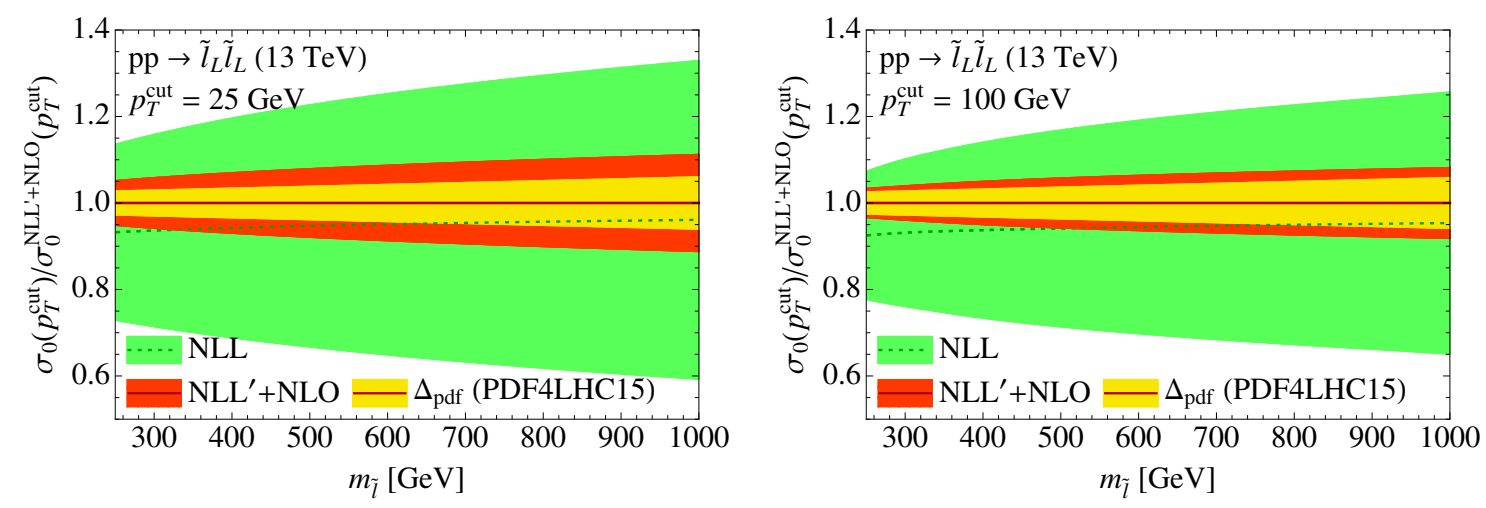

Figure 10. The 0 -jet cross section for $\tilde{\ell}_{L} \tilde{\ell}_{L}$ as a function of $m_{\tilde{\ell}}$ for $p_{T}^{\text {cut }}=25 \mathrm{GeV}$ (left) and $100 \mathrm{GeV}$ (right) at $13 \mathrm{TeV}$. The predictions are normalized to the NLL'+NLO central value. The NLL and $\mathrm{NLL}^{\prime}+\mathrm{NLO}$ perturbative uncertainty are shown by the green and orange band, respectively. The yellow band shows in addition the PDF uncertainty for the $\mathrm{NLL}^{\prime}+\mathrm{NLO}$ results, determined following ref. [86].

in ref. [86]. ${ }^{10}$ The perturbative uncertainty is still larger than the PDF uncertainty, so we are not yet limited by the latter, though they become comparable for $p_{T}^{\text {cut }}=100 \mathrm{GeV}$. In this figure, the increase of the perturbative uncertainty when going to higher slepton masses is clearly visible. For $p_{T}^{\text {cut }}=25 \mathrm{GeV}$ the relative NLL uncertainty increases from $24 \%$ at $m_{\tilde{\ell}}=300 \mathrm{GeV}$ to $38 \%$ at $m_{\tilde{\ell}}=1000 \mathrm{GeV}$. Going from NLL to NLL'+NLO, we observe a significant improvement. The $\mathrm{NLL}^{\prime}+\mathrm{NLO}$ uncertainty is roughly a factor of three to four smaller, and increases from $5.8 \%$ at $m_{\tilde{\ell}}=300 \mathrm{GeV}$ to $11.2 \%$ at $m_{\tilde{\ell}}=1000 \mathrm{GeV}$. The corresponding results for $\tilde{\ell}_{R} \tilde{\ell}_{R}$ production are very similar. Finally, we note that it is certainly feasible if necessary to further reduce the perturbative uncertainties by going one order higher to NNLL'.

\section{Conclusions}

To maximize their sensitivity, several LHC searches for new physics require a specific number of signal jets and veto additional jets with transverse momentum above a certain value $p_{T}^{\text {cut }}$, typically around $20-50 \mathrm{GeV}$. This jet veto introduces large logarithms of $p_{T}^{\text {cut }}$ over the scale of new physics in the cross section, which requires resummation to obtain the best possible predictions.

We have presented the first predictions of a SUSY cross section including the higherorder resummation of jet-veto logarithms. Focusing on slepton (selectron and smuon) production, where a 0 -jet sample is selected, we carry out resummation at NLL' order and

\footnotetext{
${ }^{10} \mathrm{An}$ alternative method to calculate the PDF uncertainties is given in eq. (24) of ref. [86]. Here the uncertainty is determined by reordering the cross sections obtained from the member PDFs and taking the spread between $68 \%$ most central ones, which is particularly suitable when the departure from the Gaussian regime is sizeable. We have checked that this method leads to slightly smaller uncertainties in our case. E.g. for $p_{T}^{\text {cut }}=25 \mathrm{GeV}$ and $m_{\tilde{\ell}}=600 \mathrm{GeV}$, the PDF uncertainty obtained from the standard deviation is $4.3 \%$, whereas the PDF uncertainty calculated with the reordering method is $4.0 \%$.
} 
match our resummed results to the NLO cross section. Here we utilize the SCET framework for jet veto resummation developed in Higgs production. Our analysis can also be extended to other new physics processes, including those with final-state jets (e.g. stop/sbottom production), which however also pose additional challenges due to the additional scales involved.

A central aspect of our study is a systematic and thorough assessment of the theory uncertainty associated with the jet veto, which we estimate using resummation profile scales. At the low resummation order provided by parton showers, this uncertainty is substantial and not accounted for in current exclusion limits quoted by ATLAS and CMS. The higher-order resummed predictions provide much improved precision and will thus benefit the interpretation of the experimental observations. One possibility to easily utilize these (and future) theoretical improvements, is for the experimental analyses to also provide results for $\sigma_{0, \text { vis }}^{95}$.

At the $13 \mathrm{TeV}$ LHC run II the slepton mass reach is expected to increase up to $\sim$ $500 \mathrm{GeV}$ and beyond with $100 \mathrm{fb}^{-1}$ (see e.g. refs. [87, 88]). Our results show that the impact of the jet veto increases further at higher slepton masses, as expected. We provide precise resummed predictions for the 0-jet slepton cross sections at $13 \mathrm{TeV}$ up to slepton masses of $1 \mathrm{TeV}$. Our predictions are available upon request. We hope that these results will allow the experimental analyses to continue relying on and benefiting from jet vetoes in optimizing the experimental sensitivity to new physics. And once discovered, accurate theory predictions will be important to reveal the nature of any new particle.

\section{Acknowledgments}

We thank Kazuki Sakurai for helpful discussions and all the ATOM authors for providing us with a version of their code. We also thank Stefan Liebler and Piotr Pietrulewicz for comments on the manuscript. This work was supported by the German Science Foundation (DFG) through the Emmy-Noether Grant No. TA 867/1-1, by the Netherlands Organization for Scientific Research (NWO) through a VENI grant, and the D-ITP consortium, a program of the NWO that is funded by the Dutch Ministry of Education, Culture and Science (OCW).

\section{A Fixed-order ingredients}

\section{A.1 Hard function}

The hard function consists of the Born cross section $\sigma_{B}$ and virtual corrections,

$$
H_{q \bar{q}}\left(Q^{2}, m_{\mathrm{SUSY}}, \mu\right)=\sigma_{B}(1+V) .
$$

The Born cross section for slepton production is (see figure 2(a))

$$
\sigma_{B}=\frac{\alpha_{\mathrm{em}}^{2} \pi}{9 Q^{2}} \frac{1}{E_{\mathrm{cm}}^{2}}\left(1-\frac{4 m_{\tilde{\ell}_{s}}^{2}}{Q^{2}}\right)^{3 / 2} h_{\tilde{\ell}_{s} \tilde{\ell}_{s}}
$$


where the index $s=L, R$ labels the slepton state. The couplings enter in

$$
h_{\tilde{\ell}_{s} \tilde{\ell}_{s}}=Q_{q}^{2} Q_{\ell}^{2}+Q_{q} Q_{\ell} \frac{\left(g_{q}^{-}+g_{q}^{+}\right)\left(g_{\ell}^{-} \delta_{s L}+g_{\ell}^{+} \delta_{s R}\right)}{1-m_{Z}^{2} / Q^{2}}+\frac{\left(g_{q}^{-2}+g_{q}^{+2}\right)\left(g_{\ell}^{-2} \delta_{s L}+g_{\ell}^{+2} \delta_{s R}\right)}{2\left(1-m_{Z}^{2} / Q^{2}\right)^{2}},
$$

where $Q_{q}$ and $Q_{\ell}$ are the electric charges, and $g_{q}^{ \pm}, g_{\ell}^{ \pm}$are the couplings to the $Z$ boson

$$
g_{f}^{-}=\frac{I_{f}^{3}-\sin ^{2} \theta_{W} Q_{f}}{\sin \theta_{W} \cos \theta_{W}}, \quad g_{f}^{+}=-\frac{\sin \theta_{W} Q_{f}}{\cos \theta_{W}},
$$

For the one-loop virtual corrections from QCD and SUSY-QCD, which are shown in figures 2(b) and 2(c), we get

$$
\begin{aligned}
V= & \frac{\alpha_{s}(\mu) C_{F}}{4 \pi}\left(V_{\mathrm{QCD}}+V_{\mathrm{SUSY}}\right)+h . c . \\
V_{\mathrm{QCD}}= & -\ln ^{2}\left(\frac{Q^{2}}{\mu^{2}}\right)+3 \ln \left(\frac{Q^{2}}{\mu^{2}}\right)-8+\frac{7 \pi^{2}}{6} \\
V_{\mathrm{SUSY}}= & 1+\frac{2 m_{\tilde{g}}^{2}-2 m_{\tilde{q}}^{2}}{Q^{2}}\left[B_{0}\left(Q^{2}, m_{\tilde{q}}^{2}, m_{\tilde{q}}^{2}\right)-B_{0}\left(0, m_{\tilde{g}}^{2}, m_{\tilde{q}}^{2}\right)\right]+B_{0}\left(Q^{2}, m_{\tilde{q}}^{2}, m_{\tilde{q}}^{2}\right) \\
& +2 \frac{m_{\tilde{g}}^{4}+\left(Q^{2}-2 m_{\tilde{q}}^{2}\right) m_{\tilde{g}}^{2}+m_{\tilde{q}}^{4}}{Q^{2}} C_{0}\left(0,0, Q^{2}, m_{\tilde{q}}^{2}, m_{\tilde{g}}^{2}, m_{\tilde{q}}^{2}\right) \\
& -B_{0}\left(0, m_{\tilde{g}}^{2}, m_{\tilde{q}}^{2}\right)+\left(m_{\tilde{q}}^{2}-m_{\tilde{g}}^{2}\right) B_{0}^{\prime}\left(0, m_{\tilde{g}}^{2}, m_{\tilde{q}}^{2}\right),
\end{aligned}
$$

where we have neglected squark mixing. This is in agreement with the expressions in refs. [45, 89-91]. $B_{0}$ and $C_{0}$ are the scalar one-loop integrals, for which we use the LoopTools conventions [92]. Note that $V_{\text {SUSY }}$ has no IR divergences in the full theory and hence does not have an explicit $\mu$ dependence and therefore cannot change the anomalous dimensions of the SCET hard function for Drell-Yan.

Since we consider a simplified model with heavy squarks and gluinos, the SUSY-QCD corrections are much smaller than the QCD corrections. In our numerical results we choose $m_{\tilde{g}}=m_{\tilde{q}}=4 \mathrm{TeV}$, though the precise value in this region is irrelevant.

\section{A.2 Beam function}

The (anti)quark beam function can be computed as a convolution of perturbative matching coefficients, $\mathcal{I}_{q j}$, and the standard PDFs, $f_{j}$,

$$
B_{q}\left(p_{T}^{\text {cut }}, x, \mu, \nu\right)=\sum_{j} \int_{x}^{1} \frac{\mathrm{d} z}{z} \mathcal{I}_{q j}\left(p_{T}^{\text {cut }}, z, \mu, \nu\right) f_{j}\left(\frac{x}{z}, \mu\right) .
$$

The matching coefficients expanded to NLO are

$$
\mathcal{I}_{q j}\left(p_{T}^{\text {cut }}, z, \mu, \nu\right)=\delta_{q j} \delta(1-z)+\frac{\alpha_{s}(\mu)}{4 \pi} \mathcal{I}_{q j}^{(1)}\left(p_{T}^{\text {cut }}, z, \mu, \nu\right)+\mathcal{O}\left(\alpha_{s}^{2}\right) .
$$

The rapidity-renormalized $\mathcal{O}\left(\alpha_{s}\right)$ matching coefficients were extracted from the calculations in ref. [25],

$$
\begin{aligned}
& \mathcal{I}_{q q}^{(1)}\left(p_{T}^{\text {cut }}, z, \mu, \nu\right)=2 C_{F}\left\{\ln \frac{\mu}{p_{T}^{\text {cut }}}\left[\left(4 \ln \frac{\nu}{Q}+3\right) \delta(1-z)-2 P_{q q}(z)\right]+I_{q q}(z)\right\}, \\
& \mathcal{I}_{q g}^{(1)}\left(p_{T}^{\text {cut }}, z, \mu, \nu\right)=2 T_{F}\left[-2 \ln \frac{\mu}{p_{T}^{\text {cut }}} P_{q g}(z)+I_{q g}(z)\right]
\end{aligned}
$$


with

$$
\begin{aligned}
P_{q q}(z) & =\left[\frac{\theta(1-z)}{1-z}\right]_{+}\left(1+z^{2}\right)+\frac{3}{2} \delta(1-z) \\
P_{q g}(z) & =\theta(1-z)\left[(1-z)^{2}+z^{2}\right] \\
I_{q q}(z) & =1-z \\
I_{q g}(z) & =2 z(1-z) .
\end{aligned}
$$

These agree with the results in refs. [22, 93, 94].

\section{A.3 Soft function}

The NLO soft function is obtained from ref. [25] using Casimir scaling

$$
S_{q \bar{q}}\left(p_{T}^{\text {cut }}, \mu, \nu\right)=1+\frac{\alpha_{s}(\mu)}{4 \pi} C_{F}\left[8 \ln \frac{\mu}{p_{T}^{\text {cut }}}\left(\ln \frac{\mu}{p_{T}^{\text {cut }}}-2 \ln \frac{\nu}{p_{T}^{\text {cut }}}\right)-\frac{\pi^{2}}{3}\right] .
$$

\section{A.4 Nonsingular contributions}

The fixed-order cross section can be split into a singular part and a nonsingular part,

$$
\sigma_{0}^{\mathrm{FO}}\left(p_{T}^{\text {cut }}\right)=\sigma_{0}^{\text {sing }}\left(p_{T}^{\text {cut }}\right)+\sigma_{0}^{\text {nons }}\left(p_{T}^{\text {cut }}\right)
$$

where we suppress the dependence on the SUSY masses for simplicity. The logarithmically enhanced terms in the singular cross section, $\sigma_{0}^{\text {sing }}\left(p_{T}^{\text {cut }}\right)$, are contained in the resummed part in eq. (2.1). The nonsingular cross section, $\sigma_{0}^{\text {nons }}\left(p_{T}^{\text {cut }}\right)$, contains terms which scale as $\mathcal{O}\left(p_{T}^{\text {cut }} / Q\right)$ and vanishes for $p_{T}^{\text {cut }} \rightarrow 0$. In this section we discuss how to extract $\sigma_{0}^{\text {nons }}\left(p_{T}^{\text {cut }}\right)$, which is essential to reproduce the correct fixed-order cross section for large $p_{T}^{\text {cut }}$.

As suggested by eq. (A.11), the NLO nonsingular cross section can be extracted from the full NLO cross section and the NLO singular cross section. We achieve this using

$$
\sigma_{0}^{\text {nons }}\left(p_{T}^{\text {cut }}\right)=\int_{\epsilon \rightarrow 0}^{p_{T}^{\text {cut }}} \mathrm{d} p_{T}^{\text {jet }}\left(\frac{\mathrm{d} \sigma_{0}^{\text {FO }}}{\mathrm{d} p_{T}^{\text {jet }}}-\frac{\mathrm{d} \sigma_{0}^{\text {sing }}}{\mathrm{d} p_{T}^{\text {jet }}}\right)
$$

The left panel of figure 11 shows the NLO results for $\mathrm{d} \sigma_{0}^{\mathrm{FO}} / \mathrm{d} p_{T}^{\mathrm{jet}}$ (red solid), $\mathrm{d} \sigma_{0}^{\mathrm{s}} / \mathrm{d} p_{T}^{\mathrm{jet}}$ (blue dashed) and their difference $\mathrm{d} \sigma_{0}^{\text {nons }} / \mathrm{d} p_{T}^{\text {jet }}$ (green dotted). We determine the NLO singular cross section, by setting all scales in the NLL' result equal to $\mu_{\mathrm{FO}}$, thus switching off the resummation. The full NLO cross section, differential in $p_{T}^{\text {jet }}$, is obtained by generating about 3 million events for $p p \rightarrow \tilde{\ell} \tilde{\ell}+j$ using Madgraph 2.3.2 [56] with a lower cutoff on $p_{T}^{\text {jet }}$ of $0.2 \mathrm{GeV}$. For small $p_{T}^{\text {jet }}$ a precise cancellation between large values of $\mathrm{d} \sigma_{0}^{\mathrm{FO}} / \mathrm{d} p_{T}^{\text {jet }}$ and $\mathrm{d} \sigma_{0}^{\mathrm{s}} / \mathrm{d} p_{T}^{\text {jet }}$ is needed to obtain a reliable result for the nonsingular cross section, see figure 11. This is achieved using a large number of Monte Carlo events and fitting the nonsingular to the functional form

$$
\frac{\mathrm{d} \sigma_{0}^{\text {nons }}}{\mathrm{d} p_{T}^{\mathrm{jet}}}=a \ln \frac{p_{T}^{\mathrm{jet}}}{2 m_{\tilde{\ell}}}+b+c \frac{p_{T}^{\mathrm{jet}}}{2 m_{\tilde{\ell}}} \ln \frac{p_{T}^{\mathrm{jet}}}{2 m_{\tilde{\ell}}}+d \frac{p_{T}^{\mathrm{jet}}}{2 m_{\tilde{\ell}}},
$$



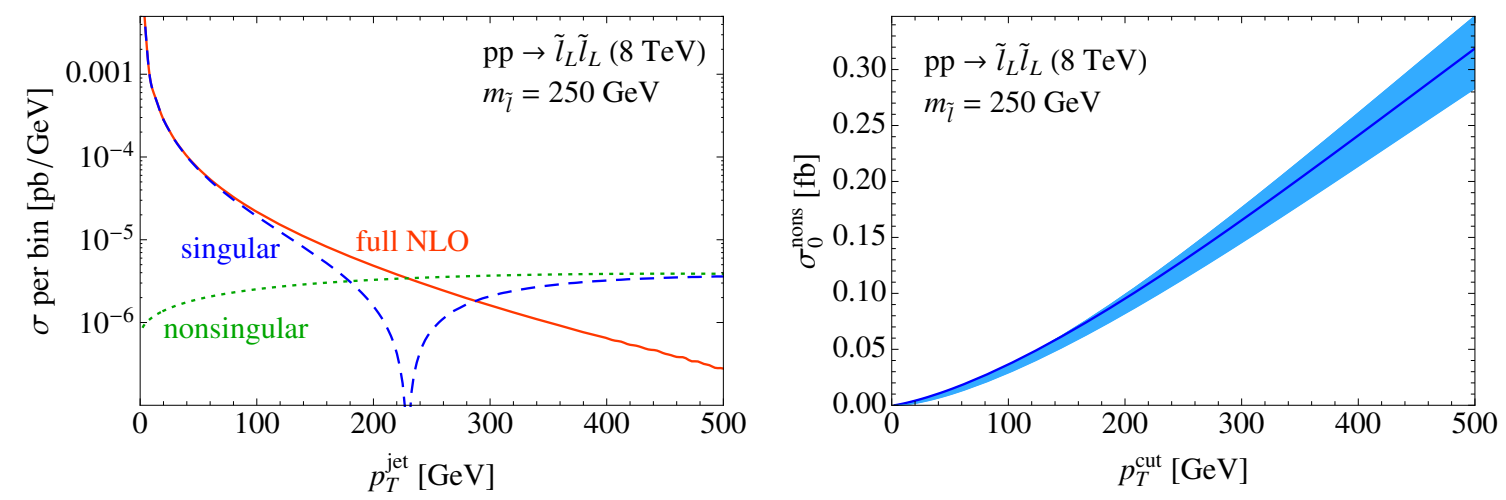

Figure 11. Left: singular (blue dashed) and nonsingular (green dotted) contributions to the full NLO (red solid) differential cross section for $\tilde{\ell}_{L} \tilde{\ell}_{L}$ production. Right: the (integrated) nonsingular cross section for $\tilde{\ell}_{L} \tilde{\ell}_{L}$ at NLO.

which has the correct leading behavior for the differential spectrum for $p_{T}^{\text {jet }} \rightarrow 0$. In this fit all points with $p_{T}^{\text {jet }}<x$ are included, where the default is $x=2 m_{\tilde{\ell}}$. As an important cross check, we ensure that the fitted result is stable under varying $x$. The left panel of figure 11 shows that for $p_{T}^{\text {jet }} \gtrsim m_{\tilde{\ell}}$, the nonsingular contributions are of the same size as the singular contributions, requiring their inclusion to correctly reproduce the full fixed-order cross section. Our final results for the NLO $\sigma_{0}^{\text {nons }}\left(p_{T}^{\text {cut }}\right)$ can be seen in the right panel of figure 11. The band indicates the perturbative uncertainty, and is obtained by calculating the nonsingular terms three times, evaluating the ingredients at $\mu_{\mathrm{FO}}=m_{\tilde{\ell}}, 2 m_{\tilde{\ell}}$ and $4 m_{\tilde{\ell}}$. The nonsingular for right-handed slepton production is obtained in the same manner.

\section{B RGE ingredients}

As explained in section 2.1, the resummation of large logarithms is achieved in SCET by first evaluating the functions in the factorized cross section eq. (2.1) at their natural virtuality $\left(\mu_{H}, \mu_{B}, \mu_{S}\right)$ and rapidity $\left(\nu_{B}, \nu_{S}\right)$ scales, and then RG evolving them to (arbitrary) common scales $\mu$ and $\nu$. Writing this evolution out explicitly, eq. (2.1) for inclusive slepton production becomes

$$
\begin{aligned}
\sigma_{0}\left(p_{T}^{\text {cut }}, m_{\mathrm{SUSY}}\right)= & \int \mathrm{d} Q^{2} \mathrm{~d} Y H_{q \bar{q}}\left(Q^{2}, m_{\mathrm{SUSY}}, \mu_{H}\right) \\
& \times B_{q}\left(p_{T}^{\text {cut }}, x_{a}, \mu_{B}, \nu_{B}\right) B_{\bar{q}}\left(p_{T}^{\text {cut }}, x_{b}, \mu_{B}, \nu_{B}\right) S_{q \bar{q}}\left(p_{T}^{\text {cut }}, \mu_{S}, \nu_{S}\right) \\
& \times U_{0}\left(p_{T}^{\text {cut }}, Q^{2} ; \mu_{H}, \mu_{B}, \mu_{S}, \nu_{B}, \nu_{S}\right)+\sigma_{0}^{\text {nons }}\left(p_{T}^{\text {cut }}, m_{\mathrm{SUSY}}\right) .
\end{aligned}
$$

At NLL' (NLL) order, we have to include the NLO (LO) results for the hard, beam and soft functions, given in appendix A. The evolution factor $U_{0}$ is given by the product of the individual evolution factors that evolve each of the functions from their natural scale to 
the common scales $\mu$ and $\nu$,

$$
\begin{aligned}
U_{0}\left(p_{T}^{\text {cut }}, Q^{2} ; \mu_{H}, \mu_{B}, \mu_{S}, \nu_{B}, \nu_{S}\right)= & \left|\exp \left[\int_{\mu_{H}}^{\mu} \frac{\mathrm{d} \mu^{\prime}}{\mu^{\prime}} \gamma_{H}^{q}\left(Q^{2}, \mu^{\prime}\right)\right]\right|^{2} \\
& \times \exp \left[\int_{\mu_{B}}^{\mu} \frac{\mathrm{d} \mu^{\prime}}{\mu^{\prime}} 2 \gamma_{B}^{q}\left(Q, \mu^{\prime}, \nu\right)\right] \exp \left[\int_{\mu_{S}}^{\mu} \frac{\mathrm{d} \mu^{\prime}}{\mu^{\prime}} \gamma_{S}^{q}\left(\mu^{\prime}, \nu\right)\right] \\
& \times \exp \left[\ln \frac{\nu_{B}}{\nu} \gamma_{\nu}^{q}\left(p_{T}^{\text {cut }}, \mu_{B}\right)+\ln \frac{\nu}{\nu_{S}} \gamma_{\nu}^{q}\left(p_{T}^{\text {cut }}, \mu_{S}\right)\right] .
\end{aligned}
$$

The anomalous dimensions entering here are collected in the next subsection. Note that due to RGE consistency the dependence on the arbitrary scales $\mu$ and $\nu$ exactly cancels between the different factors in eq. (B.2).

\section{B.1 Anomalous dimensions}

The anomalous dimension of the hard, beam, and soft functions that enter in the evolution kernel in eq. (B.2) have the following general structure [20, 89]

$$
\begin{aligned}
\gamma_{H}^{q}\left(Q^{2}, \mu\right) & =\Gamma_{\text {cusp }}^{q}\left[\alpha_{s}(\mu)\right] \ln \frac{Q^{2}}{\mu^{2}}+\gamma_{H}^{q}\left[\alpha_{s}(\mu)\right], \\
\gamma_{B}^{q}(Q, \mu, \nu) & =2 \Gamma_{\text {cusp }}^{q}\left[\alpha_{s}(\mu)\right] \ln \frac{\nu}{Q}+\gamma_{B}^{q}\left[\alpha_{s}(\mu)\right], \\
\gamma_{S}^{q}(\mu, \nu) & =4 \Gamma_{\text {cusp }}^{q}\left[\alpha_{s}(\mu)\right] \ln \frac{\mu}{\nu}+\gamma_{S}^{q}\left[\alpha_{s}(\mu)\right], \\
\gamma_{\nu}^{q}\left(p_{T}^{\text {cut }}, \mu\right) & =-4 \eta_{\Gamma}^{q}\left(p_{T}^{\text {cut }}, \mu\right)+\gamma_{\nu}^{q}\left[\alpha_{s}\left(p_{T}^{\text {cut }}\right)\right],
\end{aligned}
$$

where the exact path-independence of the evolution in $(\mu, \nu)$ space [54] is ensured by

$$
\eta_{\Gamma}^{q}\left(\mu_{0}, \mu\right)=\int_{\mu_{0}}^{\mu} \frac{\mathrm{d} \mu^{\prime}}{\mu^{\prime}} \Gamma_{\text {cusp }}^{q}\left[\alpha_{s}\left(\mu^{\prime}\right)\right] .
$$

The exact $\mu$ independence of the cross section is equivalent to the RG consistency relation

$$
2 \gamma_{H}^{q}\left(Q^{2}, \mu\right)+2 \gamma_{B}^{q}(Q, \mu, \nu)+\gamma_{S}^{q}(\mu, \nu)=0 .
$$

We give the cusp and noncusp anomalous dimensions in terms of an expansion in $\alpha_{s}$,

$$
\Gamma_{\text {cusp }}^{q}\left(\alpha_{s}\right)=\sum_{n=0}^{\infty} \Gamma_{n}^{q}\left(\frac{\alpha_{s}}{4 \pi}\right)^{n+1}, \quad \gamma_{X}^{q}\left(\alpha_{s}\right)=\sum_{n=0}^{\infty} \gamma_{X n}^{q}\left(\frac{\alpha_{s}}{4 \pi}\right)^{n+1} .
$$

At NLL (and NLL') we require the one-loop noncusp anomalous dimensions $\gamma_{X 0}^{q}$ and the two-loop cusp anomalous dimension, $\Gamma_{0}^{q}, \Gamma_{1}^{q}$, as well as the two-loop running for $\alpha_{s}$. At NNLL we would need each at one order higher, which we also give below.

The coefficients for the cusp anomalous dimension are [95, 96]

$$
\begin{aligned}
\Gamma_{0}^{q}= & 4 C_{F} \\
\Gamma_{1}^{q}= & 4 C_{F}\left[\left(\frac{67}{9}-\frac{\pi^{2}}{3}\right) C_{A}-\frac{20}{9} T_{F} n_{f}\right] \\
\Gamma_{2}^{q}= & 4 C_{F}\left[\left(\frac{245}{6}-\frac{134 \pi^{2}}{27}+\frac{11 \pi^{4}}{45}+\frac{22 \zeta_{3}}{3}\right) C_{A}^{2}+\left(-\frac{418}{27}+\frac{40 \pi^{2}}{27}-\frac{56 \zeta_{3}}{3}\right) C_{A} T_{F} n_{f}\right. \\
& \left.+\left(-\frac{55}{3}+16 \zeta_{3}\right) C_{F} T_{F} n_{f}-\frac{16}{27} T_{F}^{2} n_{f}^{2}\right] .
\end{aligned}
$$


The hard noncusp anomalous dimension is those of the quark form factor [97, 98]. The noncusp anomalous dimension coefficients for the soft function and rapidity evolution follow from ref. [25] using Casimir scaling, and those for the beam function then follow from the consistency relation in eq. (B.5). This leads to

$$
\begin{aligned}
\gamma_{H 0}^{q}= & -6 C_{F}, \\
\gamma_{H 1}^{q}= & -C_{F}\left[\left(\frac{82}{9}-52 \zeta_{3}\right) C_{A}+\left(3-4 \pi^{2}+48 \zeta_{3}\right) C_{F}+\left(\frac{65}{9}+\pi^{2}\right) \beta_{0}\right], \\
\gamma_{B 0}^{q}= & 6 C_{F} \\
\gamma_{B 1}^{q}= & C_{F}\left[\left(3-4 \pi^{2}+48 \zeta_{3}\right) C_{F}+\left(-14+16\left(1+\pi^{2}\right) \ln 2-96 \zeta_{3}\right) C_{A}\right. \\
& \left.+\left(\frac{19}{3}-\frac{4}{3} \pi^{2}+\frac{80}{3} \ln 2\right) \beta_{0}\right] \\
\gamma_{S 0}^{q}= & 0 \\
\gamma_{S 1}^{q}= & 8 C_{F}\left[\left(\frac{52}{9}-4\left(1+\pi^{2}\right) \ln 2+11 \zeta_{3}\right) C_{A}+\left(\frac{2}{9}+\frac{7 \pi^{2}}{12}-\frac{20}{3} \ln 2\right) \beta_{0}\right], \\
\gamma_{\nu 0}^{q}= & 0, \\
\gamma_{\nu 1}^{q}= & -16 C_{F}\left[\left(\frac{17}{9}-\left(1+\pi^{2}\right) \ln 2+\zeta_{3}\right) C_{A}+\left(\frac{4}{9}+\frac{\pi^{2}}{12}-\frac{5}{3} \ln 2\right) \beta_{0}\right]+C_{2}(R),
\end{aligned}
$$

where $C_{2}(R)=16 C_{F} C_{A}\left(-2.49 \ln R^{2}-0.49\right)+\mathcal{O}\left(R^{2}\right)$ denotes the clustering correction from the jet algorithm [25]. For completeness, in our convention we have

$$
\beta_{0}=\frac{11}{3} C_{A}-\frac{4}{3} T_{F} n_{f}, \quad C_{A}=N_{c}, \quad C_{F}=\frac{N_{c}^{2}-1}{2 N_{c}}=\frac{4}{3}, \quad T_{F}=\frac{1}{2},
$$

where $N_{c}=3$ is the number of colors and $n_{f}=5$ is the number of active quark flavors.

\section{B.2 Profiles scales}

In this appendix we give the expressions for the scales $\mu_{H}, \mu_{B}, \mu_{S}$ and $\nu_{B}, \nu_{S}$ employed for our central value and uncertainty estimate. A discussion of our $p_{T}$-dependent profile scales is given in section 2.3, and includes plots and our procedure for estimating the perturbative uncertainty.

At small values of $p_{T}^{\text {cut }}$ the full NLO cross section is governed by the singular cross section containing the logarithmic terms which need to be resummed; see the left panel of figure 11 and its discussion. From the anomalous dimensions in eq. (B.3) we can read off the canonical scales already given in eq. (2.4) for which the logarithms in the functions are minimized,

$$
\begin{aligned}
& \mu_{H}=2 m_{\tilde{\ell}} \sim Q, \\
& \mu_{B}=p_{T}^{\text {cut }}, \quad \nu_{B}=2 m_{\tilde{\ell}} \sim Q, \\
& \mu_{S}=p_{T}^{\text {cut }}, \quad \nu_{S}=p_{T}^{\text {cut }} .
\end{aligned}
$$

These are the appropriate scale choices in the resummation region. 
At large values $p_{T}^{\text {cut }} \sim Q$, singular and nonsingular contributions are of similar size and there are large cancellations between them. This can be observed in the left panel of figure 11 , where for $p_{T}^{\text {cut }} \gtrsim 300 \mathrm{GeV}$ the singular and nonsingular contributions have larger magnitudes (and opposite signs) than the full result. To reproduce this cancellation and thus the fixed-order result, resummation must be turned off at this point. This is achieved by evaluating all functions in the factorized cross section at a common fixed-order scale

$$
\mu_{H}=\mu_{B}=\mu_{S}=\nu_{B}=\nu_{S}=\mu_{\mathrm{FO}}=2 m_{\tilde{\ell}},
$$

which is also the scale used for the nonsingular corrections. The value $\mu_{\mathrm{FO}}=2 m_{\tilde{\ell}} \sim Q$ is chosen to agree with the value of $\mu_{H}$ used at small $p_{T}^{\text {cut }}$. In the intermediate region, both resummation and fixed order terms are relevant. In this region, the scales are chosen to smoothly interpolate between the resummation region at small $p_{T}^{\text {cut }}$ values and the fixedorder region at large $p_{T}^{\text {cut }}$ values.

We follow ref. [25] and choose our (central) profile scales according to

$$
\begin{aligned}
& \mu_{H}=\nu_{B}=\mu_{\mathrm{FO}}, \\
& \mu_{B}=\mu_{S}=\nu_{S}=\mu_{\mathrm{FO}} \times f_{\mathrm{run}}\left(p_{T}^{\text {cut }} /\left(2 m_{\tilde{\ell}}\right)\right),
\end{aligned}
$$

with

$$
f_{\text {run }}(x)=\left\{\begin{array}{lll}
x_{0}\left[1+\left(x / x_{0}\right)^{2} / 4\right] & x \leq 2 x_{0} & \text { nonperturbative region } \\
x & 2 x_{0} \leq x \leq x_{1} & \text { resummation region } \\
x+\frac{\left(2-x_{2}-x_{3}\right)\left(x-x_{1}\right)^{2}}{2\left(x_{2}-x_{1}\right)\left(x_{3}-x_{1}\right)} & x_{1} \leq x \leq x_{2} & \text { transition from resummation } \\
1-\frac{\left(2-x_{1}-x_{2}\right)\left(x-x_{3}\right)^{2}}{2\left(x_{3}-x_{1}\right)\left(x_{3}-x_{2}\right)} & x_{2} \leq x \leq x_{3} & \text { transition to fixed order } \\
1 & x_{3} \leq x & \text { fixed-order region }
\end{array}\right.
$$

The values for $x_{1}, x_{2}, x_{3}$ determine where the transition from resummation to fixed-order region happens. They are chosen as

$$
\left\{x_{1}, x_{2}, x_{3}\right\}=\{0.15,0.4,0.65\}
$$

by considering the relative size of the singular and nonsingular terms in figure 11. Below $x_{1}$ we have exact canonical running, eq. (B.10), while above $x_{3}$ the resummation is fully turned off. For $2 m_{\tilde{\ell}}=500 \mathrm{GeV}$ this corresponds to $\{75,200,325\} \mathrm{GeV}$. In addition we choose $x_{0}=2.5 \mathrm{GeV} / \mu_{\mathrm{FO}}$. The resulting central scales are shown as solid blue $\left(\mu_{H}, \nu_{B}\right)$ and red $\left(\mu_{B}, \mu_{S}, \nu_{S}\right)$ lines in figure 5.

To estimate the perturbative uncertainties in the resummed prediction, variations of the profile scales are considered, as discussed in section 2.3. Here we very briefly summarize the variations; more details on their derivation can be found in ref. [25]. The set of variations $V_{\mu}$ determining $\Delta_{\mu}$ has 14 profile scale variations, which are all possible combinations of

1. an overall up and down variation of the fixed-order scale $\mu_{\mathrm{FO}}$ by factors of 2 and $1 / 2$, 
2. four variations for the transition points $x_{1}, x_{2}, x_{3}$

$$
\left\{x_{1}, x_{2}, x_{3}\right\}:\{0.1,0.3,0.5\},\{0.2,0.5,0.8\},\{0.04,0.4,0.8\},\{0.2,0.35,0.5\} .
$$

The set of variations $V_{\text {resum }}$ of $\mu_{B}, \mu_{S}, \nu_{B}, \nu_{S}$ determining $\Delta_{\text {resum }}$ are combinations of

$$
\begin{aligned}
\mu_{i}^{\text {up }}\left(p_{T}^{\text {cut }}\right) & =\mu_{i}^{\text {central }}\left(p_{T}^{\text {cut }}\right) \times f_{\text {vary }}\left(p_{T}^{\text {cut }} /\left(2 m_{\tilde{\ell}}\right)\right), \\
\mu_{i}^{\text {down }}\left(p_{T}^{\text {cut }}\right) & =\mu_{i}^{\text {central }}\left(p_{T}^{\text {cut }}\right) / f_{\text {vary }}\left(p_{T}^{\text {cut }} /\left(2 m_{\tilde{\ell}}\right)\right), \\
\nu_{i}^{\text {up }}\left(p_{T}^{\text {cut }}\right) & =\nu_{i}^{\text {central }}\left(p_{T}^{\text {cut }}\right) \times f_{\text {vary }}\left(p_{T}^{\text {cut }} /\left(2 m_{\tilde{\ell}}\right)\right), \\
\nu_{i}^{\text {down }}\left(p_{T}^{\text {cut }}\right) & =\nu_{i}^{\text {central }}\left(p_{T}^{\text {cut }}\right) / f_{\text {vary }}\left(p_{T}^{\text {cut }} /\left(2 m_{\tilde{\ell}}\right)\right) .
\end{aligned}
$$

The multiplicative variation factor is defined as

$$
f_{\text {vary }}(x)= \begin{cases}2\left(1-x^{2} / x_{3}^{2}\right) & 0 \leq x \leq x_{3} / 2, \\ 1+2\left(1-x / x_{3}\right)^{2} & x_{3} / 2 \leq x \leq x_{3}, \\ 1 & x_{3} \leq x,\end{cases}
$$

which approaches a factor of 2 for $p_{T}^{\text {cut }} \rightarrow 0$ and turns off for $x \rightarrow x_{3}$. Out of the 80 possible combinations of variations, all combinations leading to arguments of logarithms which are more then a factor of 2 different from their central values are not considered. This leaves a total of 35 profile scale variations in $V_{\text {resum }}$.

Open Access. This article is distributed under the terms of the Creative Commons Attribution License (CC-BY 4.0), which permits any use, distribution and reproduction in any medium, provided the original author(s) and source are credited.

\section{References}

[1] ATLAS collaboration, Search for direct third-generation squark pair production in final states with missing transverse momentum and two b-jets in $\sqrt{s}=8 \mathrm{TeV} p p$ collisions with the ATLAS detector, JHEP 10 (2013) 189 [arXiv:1308.2631] [INSPIRE].

[2] CMS collaboration, Search for direct production of bottom squark pairs, CMS-PAS-SUS-13-018 (2013).

[3] ATLAS collaboration, ATLAS Run 1 searches for direct pair production of third-generation squarks at the Large Hadron Collider, Eur. Phys. J. C 75 (2015) 510 [arXiv:1506.08616] [INSPIRE].

[4] ATLAS collaboration, Search for the direct production of charginos, neutralinos and staus in final states with at least two hadronically decaying taus and missing transverse momentum in pp collisions at $\sqrt{s}=8 \mathrm{TeV}$ with the ATLAS detector, JHEP 10 (2014) 096 [arXiv: 1407.0350] [INSPIRE].

[5] ATLAS collaboration, Search for direct production of charginos, neutralinos and sleptons in final states with two leptons and missing transverse momentum in pp collisions at $\sqrt{s}=8 \mathrm{TeV}$ with the ATLAS detector, JHEP 05 (2014) 071 [arXiv: 1403.5294] [INSPIRE].

[6] CMS collaboration, Searches for electroweak production of charginos, neutralinos and sleptons decaying to leptons and $W, Z$ and Higgs bosons in pp collisions at $8 \mathrm{TeV}$, Eur. Phys. J. C 74 (2014) 3036 [arXiv:1405.7570] [INSPIRE]. 
[7] ATLAS collaboration, Search for direct pair production of a chargino and a neutralino decaying to the $125 \mathrm{GeV}$ Higgs boson in $\sqrt{s}=8 \mathrm{TeV}$ pp collisions with the ATLAS detector, Eur. Phys. J. C 75 (2015) 208 [arXiv:1501.07110] [InSPIRE].

[8] ATLAS collaboration, Search for the electroweak production of supersymmetric particles in $\sqrt{s}=8 \mathrm{TeV}$ pp collisions with the ATLAS detector, Phys. Rev. D 93 (2016) 052002 [arXiv: 1509.07152] [INSPIRE].

[9] ATLAS collaboration, Measurement of $Z Z$ production in pp collisions at $\sqrt{s}=7 \mathrm{TeV}$ and limits on anomalous $Z Z Z$ and $Z Z \gamma$ couplings with the ATLAS detector, JHEP 03 (2013) 128 [arXiv:1211.6096] [INSPIRE].

[10] CMS collaboration, Search for dark matter and unparticles produced in association with a $Z$ boson in proton-proton collisions at $\sqrt{s}=8 \mathrm{TeV}$, Phys. Rev. D 93 (2016) 052011 [arXiv: 1511.09375] [INSPIRE].

[11] ATLAS collaboration, Search for dark matter candidates and large extra dimensions in events with a photon and missing transverse momentum in pp collision data at $\sqrt{s}=7 \mathrm{TeV}$ with the ATLAS detector, Phys. Rev. Lett. 110 (2013) 011802 [arXiv:1209.4625] [INSPIRE].

[12] ATLAS collaboration, Search for dark matter in events with a $Z$ boson and missing transverse momentum in pp collisions at $\sqrt{s}=8 \mathrm{TeV}$ with the ATLAS detector, Phys. Rev. D 90 (2014) 012004 [arXiv: 1404.0051] [INSPIRE].

[13] CMS collaboration, Monophoton search, CMS-PAS-EXO-12-047 (2012).

[14] C.F. Berger, C. Marcantonini, I.W. Stewart, F.J. Tackmann and W.J. Waalewijn, Higgs Production with a Central Jet Veto at NNLL+NNLO, JHEP 04 (2011) 092 [arXiv: 1012.4480] [INSPIRE].

[15] I.W. Stewart and F.J. Tackmann, Theory Uncertainties for Higgs and Other Searches Using Jet Bins, Phys. Rev. D 85 (2012) 034011 [arXiv:1107.2117] [InSPIRE].

[16] I.W. Stewart, F.J. Tackmann and W.J. Waalewijn, Factorization at the LHC: From PDFs to Initial State Jets, Phys. Rev. D 81 (2010) 094035 [arXiv:0910.0467] [InSPIRE].

[17] I.W. Stewart, F.J. Tackmann and W.J. Waalewijn, N-Jettiness: An Inclusive Event Shape to Veto Jets, Phys. Rev. Lett. 105 (2010) 092002 [arXiv: 1004.2489] [InSPIRE].

[18] A. Banfi, G.P. Salam and G. Zanderighi, $N L L+N N L O$ predictions for jet-veto efficiencies in Higgs-boson and Drell-Yan production, JHEP 06 (2012) 159 [arXiv:1203.5773] [INSPIRE].

[19] T. Becher and M. Neubert, Factorization and NNLL Resummation for Higgs Production with a Jet Veto, JHEP 07 (2012) 108 [arXiv: 1205.3806] [INSPIRE].

[20] F.J. Tackmann, J.R. Walsh and S. Zuberi, Resummation Properties of Jet Vetoes at the LHC, Phys. Rev. D 86 (2012) 053011 [arXiv:1206.4312] [INSPIRE].

[21] A. Banfi, P.F. Monni, G.P. Salam and G. Zanderighi, Higgs and Z-boson production with a jet veto, Phys. Rev. Lett. 109 (2012) 202001 [arXiv:1206.4998] [INSPIRE].

[22] X. Liu and F. Petriello, Resummation of jet-veto logarithms in hadronic processes containing jets, Phys. Rev. D 87 (2013) 014018 [arXiv: 1210.1906] [INSPIRE].

[23] X. Liu and F. Petriello, Reducing theoretical uncertainties for exclusive Higgs-boson plus one-jet production at the LHC, Phys. Rev. D 87 (2013) 094027 [arXiv:1303.4405] [INSPIRE].

[24] T. Becher, M. Neubert and L. Rothen, Factorization and $N^{3} L L_{p}+N N L O$ predictions for the Higgs cross section with a jet veto, JHEP 10 (2013) 125 [arXiv:1307.0025] [INSPIRE]. 
[25] I.W. Stewart, F.J. Tackmann, J.R. Walsh and S. Zuberi, Jet $p_{T}$ resummation in Higgs production at $N N L L^{\prime}+N N L O$, Phys. Rev. D 89 (2014) 054001 [arXiv:1307.1808] [INSPIRE].

[26] A. Banfi, P.F. Monni and G. Zanderighi, Quark masses in Higgs production with a jet veto, JHEP 01 (2014) 097 [arXiv: 1308.4634] [INSPIRE].

[27] R. Boughezal, X. Liu, F. Petriello, F.J. Tackmann and J.R. Walsh, Combining Resummed Higgs Predictions Across Jet Bins, Phys. Rev. D 89 (2014) 074044 [arXiv:1312.4535] [INSPIRE].

[28] S. Gangal, M. Stahlhofen and F.J. Tackmann, Rapidity-Dependent Jet Vetoes, Phys. Rev. D 91 (2015) 054023 [arXiv: 1412.4792] [INSPIRE].

[29] A. Banfi et al., Jet-vetoed Higgs cross section in gluon fusion at $N^{3} L O+N N L L$ with small-R resummation, JHEP 04 (2016) 049 [arXiv: 1511.02886] [INSPIRE].

[30] D.Y. Shao, C.S. Li and H.T. Li, Resummation Prediction on Higgs and Vector Boson Associated Production with a Jet Veto at the LHC, JHEP 02 (2014) 117 [arXiv:1309.5015] [INSPIRE].

[31] Y. Li and X. Liu, High precision predictions for exclusive VH production at the LHC, JHEP 06 (2014) 028 [arXiv: 1401.2149] [INSPIRE].

[32] I. Moult and I.W. Stewart, Jet Vetoes interfering with $H \rightarrow W W$, JHEP 09 (2014) 129 [arXiv: 1405.5534] [INSPIRE].

[33] P. Jaiswal and T. Okui, Explanation of the $W W$ excess at the LHC by jet-veto resummation, Phys. Rev. D 90 (2014) 073009 [arXiv:1407.4537] [INSPIRE].

[34] T. Becher, R. Frederix, M. Neubert and L. Rothen, Automated NNLL + NLO resummation for jet-veto cross sections, Eur. Phys. J. C 75 (2015) 154 [arXiv:1412.8408] [INSPIRE].

[35] Y. Wang, C.S. Li and Z.L. Liu, Resummation prediction on gauge boson pair production with a jet veto, Phys. Rev. D 93 (2016) 094020 [arXiv: 1504.00509] [INSPIRE].

[36] M. Papucci, I.-W. Kim, K. Sakurai and A. Weiler, in preparation.

[37] M. Drees, H. Dreiner, D. Schmeier, J. Tattersall and J.S. Kim, CheckMATE: Confronting your Favourite New Physics Model with LHC Data, Comput. Phys. Commun. 187 (2015) 227 [arXiv: 1312.2591] [INSPIRE].

[38] W. Beenakker, M. Klasen, M. Krämer, T. Plehn, M. Spira and P.M. Zerwas, The Production of charginos/neutralinos and sleptons at hadron colliders, Phys. Rev. Lett. 83 (1999) 3780 [Erratum ibid. 100 (2008) 029901] [hep-ph/9906298] [INSPIRE].

[39] B. Fuks, M. Klasen, D.R. Lamprea and M. Rothering, Precision predictions for electroweak superpartner production at hadron colliders with Resummino, Eur. Phys. J. C 73 (2013) 2480 [arXiv: 1304.0790] [INSPIRE].

[40] B. Fuks, M. Klasen, D.R. Lamprea and M. Rothering, Revisiting slepton pair production at the Large Hadron Collider, JHEP 01 (2014) 168 [arXiv:1310.2621] [INSPIRE].

[41] M. Bahr et al., HERWIG++ Physics and Manual, Eur. Phys. J. C 58 (2008) 639 [arXiv: 0803.0883] [INSPIRE].

[42] B. Jager, A. von Manteuffel and S. Thier, Slepton pair production in the POWHEG BOX, JHEP 10 (2012) 130 [arXiv:1208.2953] [inSPIRE].

[43] M. Krämer et al., Supersymmetry production cross sections in pp collisions at $\sqrt{s}=7 \mathrm{TeV}$, arXiv:1206.2892 [INSPIRE]. 
[44] A. Broggio, M. Neubert and L. Vernazza, Soft-gluon resummation for slepton-pair production at hadron colliders, JHEP 05 (2012) 151 [arXiv:1111.6624] [INSPIRE].

[45] G. Bozzi, B. Fuks and M. Klasen, Threshold Resummation for Slepton-Pair Production at Hadron Colliders, Nucl. Phys. B 777 (2007) 157 [hep-ph/0701202] [INSPIRE].

[46] B. Jäger, A. von Manteuffel and S. Thier, Slepton pair production in association with a jet: NLO-QCD corrections and parton-shower effects, JHEP 02 (2015) 041 [arXiv:1410.3802] [INSPIRE].

[47] C.W. Bauer, S. Fleming and M.E. Luke, Summing Sudakov logarithms in $B \rightarrow X_{s} \gamma$ in effective field theory, Phys. Rev. D 63 (2000) 014006 [hep-ph/0005275] [INSPIRE].

[48] C.W. Bauer, S. Fleming, D. Pirjol and I.W. Stewart, An Effective field theory for collinear and soft gluons: Heavy to light decays, Phys. Rev. D 63 (2001) 114020 [hep-ph/0011336] [INSPIRE].

[49] C.W. Bauer and I.W. Stewart, Invariant operators in collinear effective theory, Phys. Lett. B 516 (2001) 134 [hep-ph/0107001] [INSPIRE].

[50] C.W. Bauer, D. Pirjol and I.W. Stewart, Soft collinear factorization in effective field theory, Phys. Rev. D 65 (2002) 054022 [hep-ph/0109045] [INSPIRE].

[51] C.W. Bauer, S. Fleming, D. Pirjol, I.Z. Rothstein and I.W. Stewart, Hard scattering factorization from effective field theory, Phys. Rev. D 66 (2002) 014017 [hep-ph/0202088] [INSPIRE].

[52] M. Beneke, A.P. Chapovsky, M. Diehl and T. Feldmann, Soft collinear effective theory and heavy to light currents beyond leading power, Nucl. Phys. B 643 (2002) 431 [hep-ph/0206152] [INSPIRE].

[53] J.-y. Chiu, A. Jain, D. Neill and I.Z. Rothstein, The Rapidity Renormalization Group, Phys. Rev. Lett. 108 (2012) 151601 [arXiv:1104.0881] [InSPIRE].

[54] J.-Y. Chiu, A. Jain, D. Neill and I.Z. Rothstein, A Formalism for the Systematic Treatment of Rapidity Logarithms in Quantum Field Theory, JHEP 05 (2012) 084 [arXiv:1202.0814] [INSPIRE].

[55] M. Procura, W.J. Waalewijn and L. Zeune, Resummation of Double-Differential Cross Sections and Fully-Unintegrated Parton Distribution Functions, JHEP 02 (2015) 117 [arXiv: 1410.6483] [INSPIRE].

[56] J. Alwall et al., The automated computation of tree-level and next-to-leading order differential cross sections and their matching to parton shower simulations, JHEP 07 (2014) 079 [arXiv: 1405.0301] [INSPIRE].

[57] C.G. Lester and D.J. Summers, Measuring masses of semiinvisibly decaying particles pair produced at hadron colliders, Phys. Lett. B 463 (1999) 99 [hep-ph/9906349] [INSPIRE].

[58] A. Barr, C. Lester and P. Stephens, m(T2): The Truth behind the glamour, J. Phys. G 29 (2003) 2343 [hep-ph/0304226] [INSPIRE].

[59] S. Dawson, E. Eichten and C. Quigg, Search for Supersymmetric Particles in Hadron-Hadron Collisions, Phys. Rev. D 31 (1985) 1581 [inSPIRE].

[60] P. Chiappetta, J. Soffer and P. Taxil, Spin Asymmetries for Scalar Leptons From $W$ and $Z$ Decay in P $\bar{P}$ Collisions, Phys. Lett. B 162 (1985) 192 [INSPIRE].

[61] F. del Aguila and L. Ametller, On the detectability of sleptons at large hadron colliders, Phys. Lett. B 261 (1991) 326 [INSPIRE]. 
[62] H. Baer, C.-h. Chen, F. Paige and X. Tata, Detecting Sleptons at Hadron Colliders and Supercolliders, Phys. Rev. D 49 (1994) 3283 [hep-ph/9311248] [INSPIRE].

[63] G. Altarelli, R.K. Ellis and G. Martinelli, Large Perturbative Corrections to the Drell-Yan Process in QCD, Nucl. Phys. B 157 (1979) 461 [InSPIRE].

[64] G. Bozzi, B. Fuks and M. Klasen, Slepton production in polarized hadron collisions, Phys. Lett. B 609 (2005) 339 [hep-ph/0411318] [INSPIRE].

[65] M. Bisset, S. Raychaudhuri and P. Roy, Higgs mediated slepton pair production at the large hadron collider, hep-ph/9602430 [INSPIRE].

[66] F. Borzumati and K. Hagiwara, Testing supersymmetry at the LHC through gluon-fusion production of a slepton pair, JHEP 03 (2011) 103 [arXiv:0912.0454] [INSPIRE].

[67] Z. Ligeti, I.W. Stewart and F.J. Tackmann, Treating the $b$ quark distribution function with reliable uncertainties, Phys. Rev. D 78 (2008) 114014 [arXiv:0807.1926] [INSPIRE].

[68] R. Abbate, M. Fickinger, A.H. Hoang, V. Mateu and I.W. Stewart, Thrust at $N^{3} L L$ with Power Corrections and a Precision Global Fit for alpha $a_{s}\left(m_{Z}\right)$, Phys. Rev. D 83 (2011) 074021 [arXiv: 1006 . 3080] [INSPIRE].

[69] A. Jain, M. Procura, B. Shotwell and W.J. Waalewijn, Fragmentation with a Cut on Thrust: Predictions for B-factories, Phys. Rev. D 87 (2013) 074013 [arXiv:1207.4788] [INSPIRE].

[70] T.T. Jouttenus, I.W. Stewart, F.J. Tackmann and W.J. Waalewijn, Jet mass spectra in Higgs boson plus one jet at next-to-next-to-leading logarithmic order, Phys. Rev. D 88 (2013) 054031 [arXiv: 1302 .0846] [INSPIRE].

[71] D. Kang, C. Lee and I.W. Stewart, Using 1-Jettiness to Measure 2 Jets in DIS 3 Ways, Phys. Rev. D 88 (2013) 054004 [arXiv:1303.6952] [INSPIRE].

[72] Z.-B. Kang, X. Liu and S. Mantry, 1-jettiness DIS event shape: NNLL+NLO results, Phys. Rev. D 90 (2014) 014041 [arXiv: 1312.0301] [INSPIRE].

[73] A.J. Larkoski, D. Neill and J. Thaler, Jet Shapes with the Broadening Axis, JHEP 04 (2014) 017 [arXiv: 1401.2158$]$ [INSPIRE].

[74] P. Pietrulewicz, S. Gritschacher, A.H. Hoang, I. Jemos and V. Mateu, Variable Flavor Number Scheme for Final State Jets in Thrust, Phys. Rev. D 90 (2014) 114001 [arXiv: 1405.4860] [INSPIRE].

[75] D. Neill, I.Z. Rothstein and V. Vaidya, The Higgs Transverse Momentum Distribution at NNLL and its Theoretical Errors, JHEP 12 (2015) 097 [arXiv: 1503.00005] [INSPIRE].

[76] S. Alioli, C.W. Bauer, C. Berggren, F.J. Tackmann and J.R. Walsh, Drell-Yan production at NNLL'+NNLO matched to parton showers, Phys. Rev. D 92 (2015) 094020 [arXiv: 1508.01475] [INSPIRE].

[77] M. Bonvini, A.S. Papanastasiou and F.J. Tackmann, Resummation and matching of b-quark

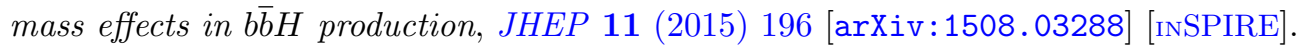

[78] A. Hornig, Y. Makris and T. Mehen, Jet Shapes in Dijet Events at the LHC in SCET, JHEP 04 (2016) 097 [arXiv: 1601.01319] [INSPIRE].

[79] J. Pumplin, D.R. Stump, J. Huston, H.L. Lai, P.M. Nadolsky and W.K. Tung, New generation of parton distributions with uncertainties from global QCD analysis, JHEP 07 (2002) 012 [hep-ph/0201195] [INSPIRE].

[80] M. Cacciari, G.P. Salam and G. Soyez, FastJet User Manual, Eur. Phys. J. C 72 (2012) 1896 [arXiv: 1111.6097] [INSPIRE]. 
[81] H.-C. Cheng and Z. Han, Minimal Kinematic Constraints and $m_{T 2}$, JHEP 12 (2008) 063 [arXiv:0810.5178] [INSPIRE].

[82] Y. Bai, H.-C. Cheng, J. Gallicchio and J. Gu, Stop the Top Background of the Stop Search, JHEP 07 (2012) 110 [arXiv: 1203.4813] [INSPIRE].

[83] DELPHES 3 collaboration, J. de Favereau et al., DELPHES 3, A modular framework for fast simulation of a generic collider experiment, JHEP 02 (2014) 057 [arXiv:1307.6346] [INSPIRE].

[84] A. Buckley et al., Rivet user manual, Comput. Phys. Commun. 184 (2013) 2803 [arXiv: 1003.0694] [INSPIRE].

[85] M. Papucci, K. Sakurai, A. Weiler and L. Zeune, Fastlim: a fast LHC limit calculator, Eur. Phys. J. C 74 (2014) 3163 [arXiv: 1402.0492] [inSPIRE].

[86] J. Butterworth et al., PDF 4 LHC recommendations for LHC Run II, J. Phys. G 43 (2016) 023001 [arXiv: 1510.03865 ] [INSPIRE].

[87] J. Eckel, M.J. Ramsey-Musolf, W. Shepherd and S. Su, Impact of LSP Character on Slepton Reach at the LHC, JHEP 11 (2014) 117 [arXiv:1408.2841] [INSPIRE].

[88] Y. Gershtein et al., Working Group Report: New Particles, Forces and Dimensions, arXiv: 1311.0299 [INSPIRE].

[89] A.V. Manohar, Deep inelastic scattering as $x \rightarrow 1$ using soft collinear effective theory, Phys. Rev. D 68 (2003) 114019 [hep-ph/0309176] [INSPIRE].

[90] C.W. Bauer, C. Lee, A.V. Manohar and M.B. Wise, Enhanced nonperturbative effects in Z decays to hadrons, Phys. Rev. D 70 (2004) 034014 [hep-ph/0309278] [INSPIRE].

[91] A. Djouadi and M. Spira, SUSY-QCD corrections to Higgs boson production at hadron colliders, Phys. Rev. D 62 (2000) 014004 [hep-ph/9912476] [InSPIRE].

[92] T. Hahn and M. Pérez-Victoria, Automatized one loop calculations in four-dimensions and D-dimensions, Comput. Phys. Commun. 118 (1999) 153 [hep-ph/9807565] [InSPIRE].

[93] M. Ritzmann and W.J. Waalewijn, Fragmentation in Jets at NNLO, Phys. Rev. D 90 (2014) 054029 [arXiv: 1407.3272 ] [INSPIRE].

[94] T. Lübbert, J. Oredsson and M. Stahlhofen, Rapidity renormalized TMD soft and beam functions at two loops, JHEP 03 (2016) 168 [arXiv:1602.01829] [INSPIRE].

[95] G.P. Korchemsky and A.V. Radyushkin, Renormalization of the Wilson Loops Beyond the Leading Order, Nucl. Phys. B 283 (1987) 342 [INSPIRE].

[96] S. Moch, J.A.M. Vermaseren and A. Vogt, The three loop splitting functions in QCD: The Nonsinglet case, Nucl. Phys. B 688 (2004) 101 [hep-ph/0403192] [InSPIRE].

[97] G. Kramer and B. Lampe, Two Jet Cross-Section in $e^{+} e^{-}$Annihilation, Z. Phys. C 34 (1987) 497 [Erratum ibid. C 42 (1989) 504] [INSPIRE].

[98] T. Matsuura, S.C. van der Marck and W.L. van Neerven, The Calculation of the Second Order Soft and Virtual Contributions to the Drell-Yan Cross-Section, Nucl. Phys. B 319 (1989) 570 [INSPIRE]. 